OPEN ACCESS

Edited by:

Xin Qi,

Case Western Reserve University,

United States

Reviewed by:

Alfredo Criollo,

Universidad de Chile, Chile

Cheng Han,

Albert Einstein College of Medicine,

United States

Bilikere S. Dwarakanath,

Shanghai Proton and Heavy lon

Center (SPHIC), China

*Correspondence:

Tae-Cheon Kang

tckang@hallym.ac.kr

Received: 07 June 2018 Accepted: 17 August 2018 Published: 05 September 2018

Citation: Kim J-E and Kang T-C (2018) Differential Roles of Mitochondrial Translocation of Active Caspase-3 and HMGB1 in Neuronal Death Induced by Status Epilepticus.

Front. Cell. Neurosci. 12:301. doi: 10.3389/fncel.2018.00301

\section{Differential Roles of Mitochondrial Translocation of Active Caspase-3 and HMGB1 in Neuronal Death Induced by Status Epilepticus}

\author{
Ji-Eun Kim and Tae-Cheon Kang* \\ Department of Anatomy and Neurobiology, Institute of Epilepsy Research, College of Medicine, Hallym University, \\ Chuncheon, South Korea
}

Under pathophysiological conditions, aberrant mitochondrial dynamics lead to the different types of neuronal death: excessive mitochondrial fission provokes apoptosis and abnormal mitochondrial elongation induces necrosis. However, the underlying mechanisms how the different mitochondrial dynamics result in the distinct neuronal death patterns have been elusive. In the present study, status epilepticus (SE) evoked excessive mitochondrial fission in parvalbumin (PV) cells (one of GABAergic interneurons) and abnormal mitochondrial elongation in CA1 neurons in the rat hippocampus. These impaired mitochondrial dynamics were accompanied by mitochondrial translocations of active caspase-3 and high mobility group box 1 (HMGB1) in PV cells and CA1 neurons, respectively. WY14643 (an activator of mitochondrial fission) aggravated SE-induced PV cell loss by enhancing active caspase- 3 induction and its mitochondrial translocation, which were attenuated by Mdivi-1 (an inhibitor of mitochondrial fission). Mitochondrial HMGB1 import was not observed in PV cell. In contrast to PV cells, Mdivi-1 deteriorated SE-induced CA1 neuronal death concomitant with mitochondrial HMGB1 translocation, which was abrogated by WY14643. These findings suggest that SE-induced aberrant mitochondrial dynamics may be involved in translocation of active caspase-3 and HMGB1 into mitochondria, which regulate neuronal apoptosis and necrosis, respectively.

Keywords: epilepsy, Mdivi-1, mitochondrial fission, mitochondrial fusion, parvalbumin, seizure, WY14643

\section{INTRODUCTION}

Mitochondria play an essential role in bioenergetics and respiratory functions for cell viability. Furthermore, mitochondria are dynamic organelles, which continuously change their morphologies (referred to as dynamics) to maintain their functionality in response to extra- and intracellular circumstances by two counteracting processes of fusion (elongation) and fission (fragmentation). Furthermore, imbalance of mitochondrial dynamics lead to the distinct neuronal death under stressful conditions (Youle and Karbowski, 2005; Parone et al., 2008; DuBoff et al., 2012; Kim et al., 2014; Kim and Kang, 2017): Excessive mitochondrial fission provokes the impaired mitochondrial function triggering apoptosis. In contrast, improper segregations and localizations of mitochondria induced by aberrant mitochondrial elongation lead to necrosis (Parone et al., 2008; DuBoff et al., 2012; Kageyama et al., 2012). 
In the hippocampus, dentate hilar neurons and CA1-3 pyramidal cells are extremely vulnerable to status epilepticus (SE, a prolonged seizure activity), although dentate granule cells are resistant. Furthermore, SE-induced neuronal death shows the heterogeneous patterns (Ordy et al., 1993; Mathern et al., 1995; Wittner et al., 2001; Kim et al., 2011; Ryu et al., 2011). Briefly, SE leads to programed necrosis in CA1 neurons (Kim et al., 2014; Ko et al., 2015; Hyun et al., 2016), and caspase-3 dependent apoptosis in loss of parvalbumin (PV) cell (one of GABAergic fast-firing interneurons in the dentate gyrus; Kang et al., 2006; Kim and Kang, 2017). Interestingly, the properties of mitochondrial dynamics in response to SE are also distinct from each neuronal subpopulation. SE provokes aberrant mitochondrial elongation in CA1 neurons (Kim et al., 2014; Hyun et al., 2016), but it propels excessive mitochondrial fragmentation in PV cell (Kim and Kang, 2017). However, the underlying mechanisms how the different mitochondrial dynamics lead to the distinct neuronal death patterns have been elusive.

High mobility group box 1 (HMGB1, a non-histone DNA-binding protein), is immediately released from the nucleus to the cytoplasm undergoing necrosis and involved in giant mitochondrial formation (Scaffidi et al., 2002; Faraco et al., 2007; Gdynia et al., 2010). Furthermore, mitochondrial HMGB1 transport facilitates and deteriorates programed necrotic CA1 neuronal death induced by SE (Hyun et al., 2016). Similar to HMGB1, active (cleaved) caspase-3 translocate from cytosol to mitochondria and disintegrates mitochondrial functions during apoptosis (Chandra and Tang, 2003). Therefore, pertinent questions are raised whether mitochondrial dynamics influence mitochondrial translocations of active caspase- 3 and HMGB1, and whether their mitochondrial translocations are practically involved in the distinct neuronal death induced by SE.

Here, we demonstrate that under physiological condition mitochondrial fission was required for caspase-3 activation. Subsequently, active caspase- 3 penetrated into mitochondria. Following SE, mitochondrial translocations of active caspase-3 were restricted to PV cells, and facilitated PV cell loss. However, HMGB1 preferentially permeated into elongated mitochondria in CA1 neurons following SE. Therefore, our findings suggest that mitochondrial imports of active caspase- 3 and HMGB1 may be involved in the abnormal mitochondrial machinery-mediated neuronal death induced by SE.

\section{MATERIALS AND METHODS}

\section{Experimental Animals and Chemicals}

Male Sprague-Dawley (SD) rats (7 weeks old) were used in the present study. Animals were kept under controlled environmental conditions $\left(23-25^{\circ} \mathrm{C}, 12 \mathrm{~h}\right.$ light/dark cycle). Rats freely accessed to water and standard laboratory food during the experiment. All animal protocols were approved by the Administrative Panel on Laboratory Animal Care of Hallym University (the authorization number of the IACUC, Hallym 2018-2). All possible efforts were taken to avoid animals' suffering and to minimize the number of animals used during the experiment. All reagents were obtained from Sigma-Aldrich (St. Louis, MO, USA), except as noted.

\section{Intracerebroventricular Infusion}

Under Isoflurane anesthesia (3\% induction, 1.5\%-2\% for surgery and $1.5 \%$ maintenance in a $65: 35$ mixture of $\mathrm{N}_{2} \mathrm{O}: \mathrm{O}_{2}$ ), animals were implanted a brain infusion kit 1 (Alzet, Cupertino, CA, USA) into the right lateral ventricle on the stereotaxic frame ( $1 \mathrm{~mm}$ posterior; $1.5 \mathrm{~mm}$ lateral; $3.5 \mathrm{~mm}$ depth to the Bregma). The infusion kit was sealed with dental cement and connected to an osmotic pump (1007D, Alzet, Cupertino, CA, USA). Each osmotic pump contained: (1) vehicle; (2) Mdivi-1 (50 $\mu \mathrm{M})$ or (3) WY14643 (150 $\mu \mathrm{M})$. An osmotic pump was placed in a subcutaneous pocket between scapulas. In our previous studies (Kim et al., 2014; Kim and Kang, 2017), the concentration of each compound could not affect the seizure activity in response to pilocarpine.

\section{SE Induction}

Three days after surgery, $\mathrm{LiCl}$ ( $3 \mathrm{mEq} / \mathrm{kg}$, i.p.) was administrated to all rats $24 \mathrm{~h}$ prior to pilocarpine hydrochloride treatment (30 mg/kg, i.p.). Twenty minutes before pilocarpine or saline (control), rats were given atropine methylbromide $(5 \mathrm{mg} / \mathrm{kg}$, i.p.) to inhibit peripheral effects of pilocarpine. Two hours after SE onset, diazepam (Valium; Hoffman la Roche, Neuilly sur-Seine, France; $10 \mathrm{mg} / \mathrm{kg}$, i.p.) was administered and repeated, as needed.

\section{Tissue Processing}

At the designated time points (control, $6 \mathrm{~h}, 12 \mathrm{~h}$ and 3 days after SE induction), animals were anesthetized with urethane $(1.5 \mathrm{~g} / \mathrm{kg}$, i.p.), and transcardially perfused with phosphate-buffered saline (PBS) followed by $4 \%$ paraformaldehyde (in $0.1 \mathrm{M}$ phosphate buffer; $\mathrm{pH}$ 7.4). The brains were removed and post-fixed by the same solution for $4 \mathrm{~h}$ at $4^{\circ} \mathrm{C}$, and submerged overnight in $30 \%$ sucrose in $0.1 \mathrm{M}$ PBS for cryoprotection. The $30 \mu \mathrm{m}$-thick coronal sections were made with a cryostat, and contained in six-well plates containing PBS. During sections, we confirmed the intracerebroventricular location of a brain infusion kit. Only animals showing the exact position were used in the present study.

\section{Immunohistochemistry and Fluoro-Jade B Staining}

After incubation with $10 \%$ normal goat serum (Vector, Burlingame, CA, USA), section were reacted in the mixture of primary antibodies listed in Table 1 (in PBS containing $0.3 \%$ triton X-100) at room temperature for overnight. After washing, sections were incubated for $1 \mathrm{~h}$ in a FITC (green)-, Cy3 (red)or AMCA (blue)-conjugated secondary antibodies (Vector, Burlingame, CA, USA). For negative control, tissues were reacted with pre-immune serum instead of primary antibody. Negative control tissues did not show any immunoreactivity for primary antibody (data not shown). To analyze the neuronal damage, Fluoro-Jade B (FJB) staining was performed according to the manufacturer's instructions. Immunoreactivities were observed 
TABLE 1 | Primary antibodies used in the present study.

\begin{tabular}{|c|c|c|c|}
\hline Antigen & Host & Manufacturer (catalog number) & Dilution used \\
\hline Mitochondrial marker (Mitochondrial complex IV subunit 1, MTCO1) & Mouse & Abcam (\#ab14705) & $1: 500$ \\
\hline Active (cleaved) caspase-3 & Rabbit & Cell signaling (\#9664) & $1: 400$ \\
\hline HMGB1 & Rabbit & Abcam (\#ab18256) & $1: 100$ \\
\hline PV & Goat & Swant (\#PVG213) & $1: 100,000$ \\
\hline
\end{tabular}

using an AxioImage M2 microscope or a confocal laser scanning microscope (LSM 710, Carl Zeiss Inc., Oberkochen, Germany).

\section{Cell Count and Measurement of Mitochondrial Length and Mitochondrial HMGB1 and Active Caspase-3 Intensities}

Areas of interest $\left(1 \times 10^{5} \mu \mathrm{m}^{2}\right)$ were selected in the captured images of the dentate gyrus and the CA1 region of the hippocampus proper (15 sections per each animal), and cell numbers were counted. The number of FJB-positive neurons was also counted by the same methods. For measurement of mitochondrial length, 25 serial images (z-stack, $1 \mu \mathrm{m}$ ) were obtained from each hippocampal section and were used for 3D-reconstruction by using ZEN lite software (Blue Edition, Carl Zeiss Inc., Oberkochen, Germany). Thereafter, we measured individual mitochondrion length (long-axis) and intensities of mitochondrial HMGB1 and active caspase-3 in PV cells and CA1 neurons ( $n=20$ /section; Ko et al., 2016; Kim and Kang, 2017).

\section{Quantification of Data and Statistical Analysis}

Student's $t$-test or ANOVA were used to analyze statistical significance. Bonferroni's test was applied for post hoc comparisons. A $p$-value below 0.05 was considered statistically significant.

\section{RESULTS}

\section{SE-Induced Mitochondrial Caspase-3 Transport in PV Cell, Not CA1 Neurons}

Since SE leads to PV cell apoptosis via excessive mitochondrial fission (Kim and Kang, 2017), we first investigated the relevance between mitochondrial dynamics and mitochondrial active caspase- 3 transports. In control animals, mitochondrial length was $\sim 1.25 \mu \mathrm{m}$ in $\mathrm{PV}$ cells (Figures 1A,B). Active (cleaved) caspase-3 signal was not detected in mitochondria (Figures 1C,D). Six hours after SE, mitochondrial length in PV cells was reduced to $\sim 0.29 \mu \mathrm{m}$ ( $p<0.05$ vs. control; Figures 1A,B) and $\sim 65 \%$ of total mitochondria showed active caspase- 3 signals ( $p<0.05$ vs. control; Figures 1C,D). In contrast to PV cells, SE results in aberrant mitochondrial elongation in CA1 neurons (Kim et al., 2014; Hyun et al., 2016). Consistent with these reports, mitochondrial length was increased to $\sim 3.29 \mu \mathrm{m}$ in CA1 neurons 3 days after SE ( $p<0.05$ vs. control; Figures 2A,B). In addition, elongated mitochondria did not show active caspase-3 signals (Figures 2A,C). These findings indicate that SE-induced mitochondrial fragmentation may be accompanied by mitochondrial active caspase- 3 translocation in PV cells, but not CA1 neurons.

\section{Mitochondrial Fragmentation Is Required for Active Caspase-3 Import to Mitochondria}

Under physiological condition, mitochondrial fission is involved in a normal rate of cytochrome $c$ release (Ishihara et al., 2009). In addition, mitochondrial fragmentation is an early apoptotic event, occurring before caspase activation (Suen et al., 2008). Thus, inhibition of mitochondrial fragmentation prevents release of cytochrome $c$ and activation of caspase-mediated signaling pathway (Frank et al., 2001; Breckenridge et al., 2003; Lee et al., 2004; Germain et al., 2005; Barsoum et al., 2006). Indeed, we have reported that Mdivi-1 (a mitochondrial fission inhibitor) attenuates SE-induced PV cell apoptosis. Therefore, it is likely that mitochondrial fission may modulate mitochondrial active caspase-3 import leading to PV cell apoptosis. To confirm this hypothesis, we validated whether the regulations of mitochondrial dynamics influence mitochondrial active caspase- 3 translocation in PV cells and CA1 neurons.

In control animals, Mdivi-1 effectively elongated mitochondrial length to $>3 \mu \mathrm{m}$ in PV cells. Following SE, it prevented mitochondrial fragmentation, active caspase-3 induction and its mitochondrial translocation in $\mathrm{PV}$ cells $(p<0.05$ vs. vehicle; Figures 3A-C). Although Mdivi-1 did not induce PV cell viability under normal condition, it mitigated SE-induced PV cell loss $12 \mathrm{~h}$ after SE ( $p<0.05$ vs. vehicle; Figures 3D,E).

In contrast to Mdivi-1, WY14643 (an activator of mitochondrial fission; Lundgren et al., 1990; Zolezzi et al., 2013; Kim et al., 2014) led to mitochondrial fragmentation in PV cells under normal condition. In addition, it resulted in mitochondrial active caspase- 3 transports in $\sim 24 \%$ of total mitochondria ( $p<0.05$ vs. vehicle; Figures 4A-C). Following SE, WY14643 facilitated active caspase-3 translocation into mitochondria, thus $\sim 80 \%$ of total mitochondria showed active caspase- 3 signals ( $p<0.05$ vs. vehicle; Figures $4 \mathrm{~A}, \mathrm{C}$ ). Furthermore, WY14643 deteriorated SE-induced PV cell loss $12 \mathrm{~h}$ after SE ( $p<0.05$ vs. vehicle; Figures 4D,E), while it did not induce PV cell loss under normal condition.

Similar to PV cells, Mdivi-1 increased mitochondrial length in CA1 neurons of control- and post-SE animals ( $p<0.05$ vs. vehicle; Figures 5A,B). Mdivi-1 did not result in active caspase-3 induction and its mitochondrial translocation in CA1 neurons (Figures 5A,C). Although Mdivi-1 did not lead to CA1 neuronal death, it exacerbated SE-induced CA1 neuron degeneration 3 days after SE ( $p<0.05$ vs. vehicle; Figures 5D,E). 


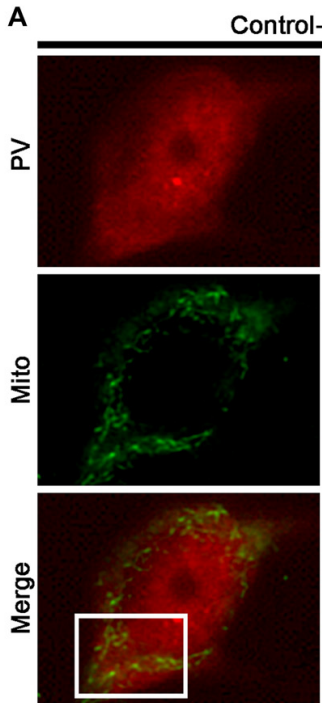

B

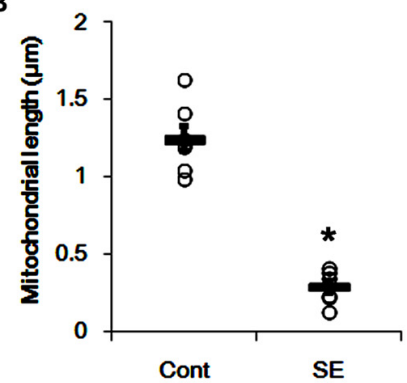

D

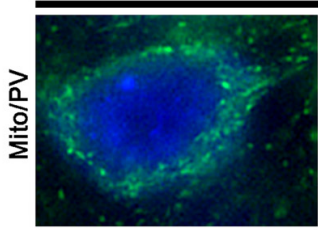

Control-Vehicle

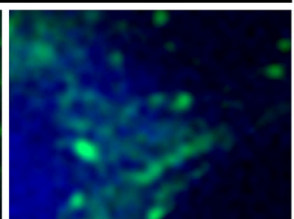

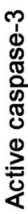
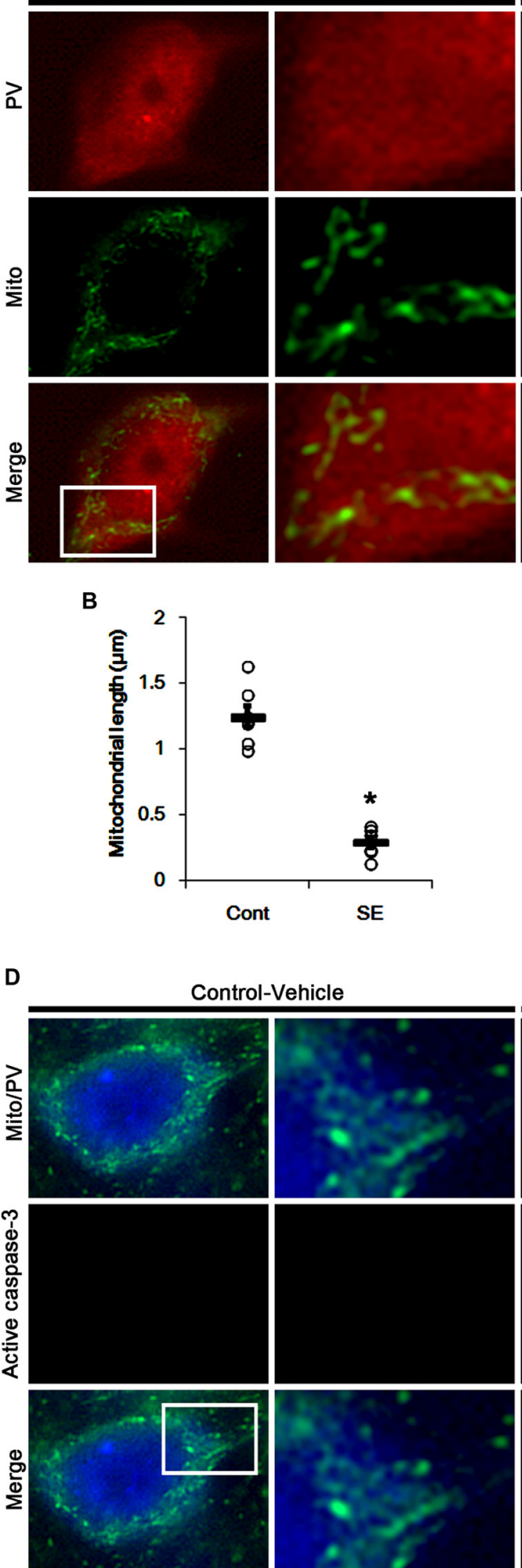

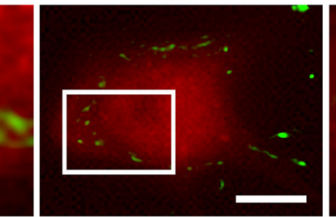

C

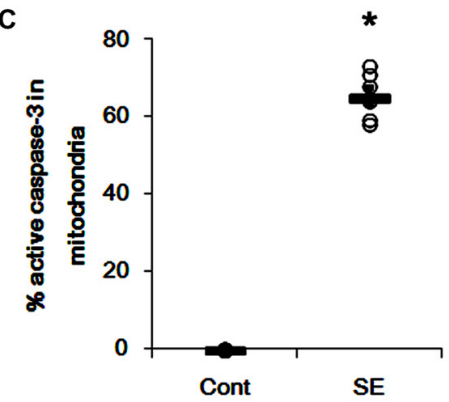

SE-Vehicle

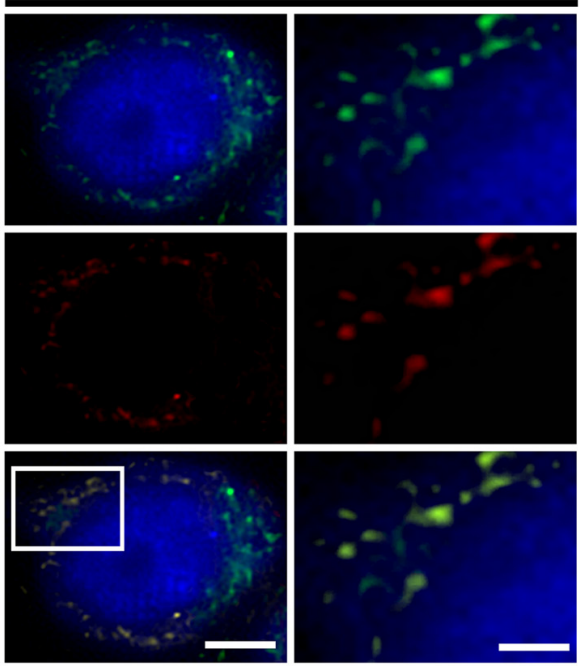

FIGURE 1 | Excessive mitochondrial fission and active caspase-3 induction in parvalbumin (PV) cells 6 h after status epilepticus (SE). SE rapidly induces mitochondrial fragmentation and translocation of active capase-3 to mitochondria in PV cells of vehicle-treated animals. (A) Representative photos of mitochondria (Mito, green) in PV (red) cells. Rectangles in merge panels (left) indicate the zoom areas for the high magnification photos (right). Bar = 5 (left panels) and 1.5 (right panels) $\mu \mathrm{m}$. (B,C) Quantification of the mitochondrial length in PV cells (B) and the fraction of active caspase-3 positive mitochondria in total mitochondria (C) in PV cells following SE. Open circles indicate each individual value. Horizontal bars indicate mean value. Error bars indicate SEM $\left({ }^{*} p<0.05\right.$ vs. control animals (Cont); $n=7$, respectively). (D) Representative photos of active caspase-3 (red) positive mitochondria (Mito, green) in PV (blue) cells. Rectangles in merge panels (left) indicate the zoom areas for the high magnification photos (right). Bar $=5$ (left panels) and 1.5 (right panels) $\mu \mathrm{m}$. 
A

CA1
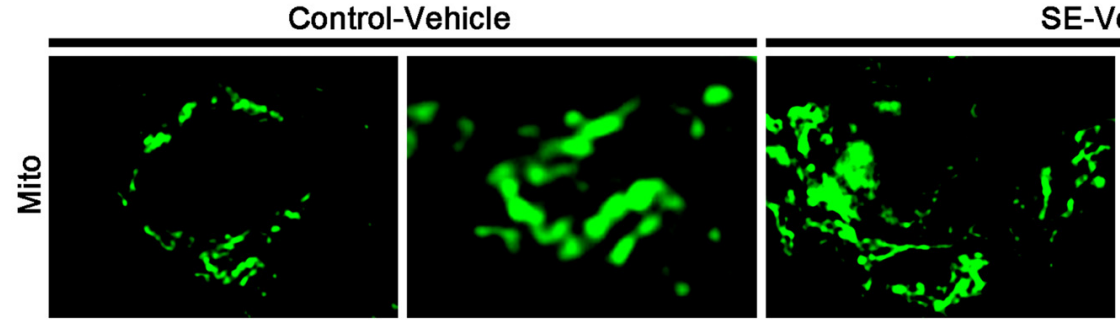

SE-Vehicle
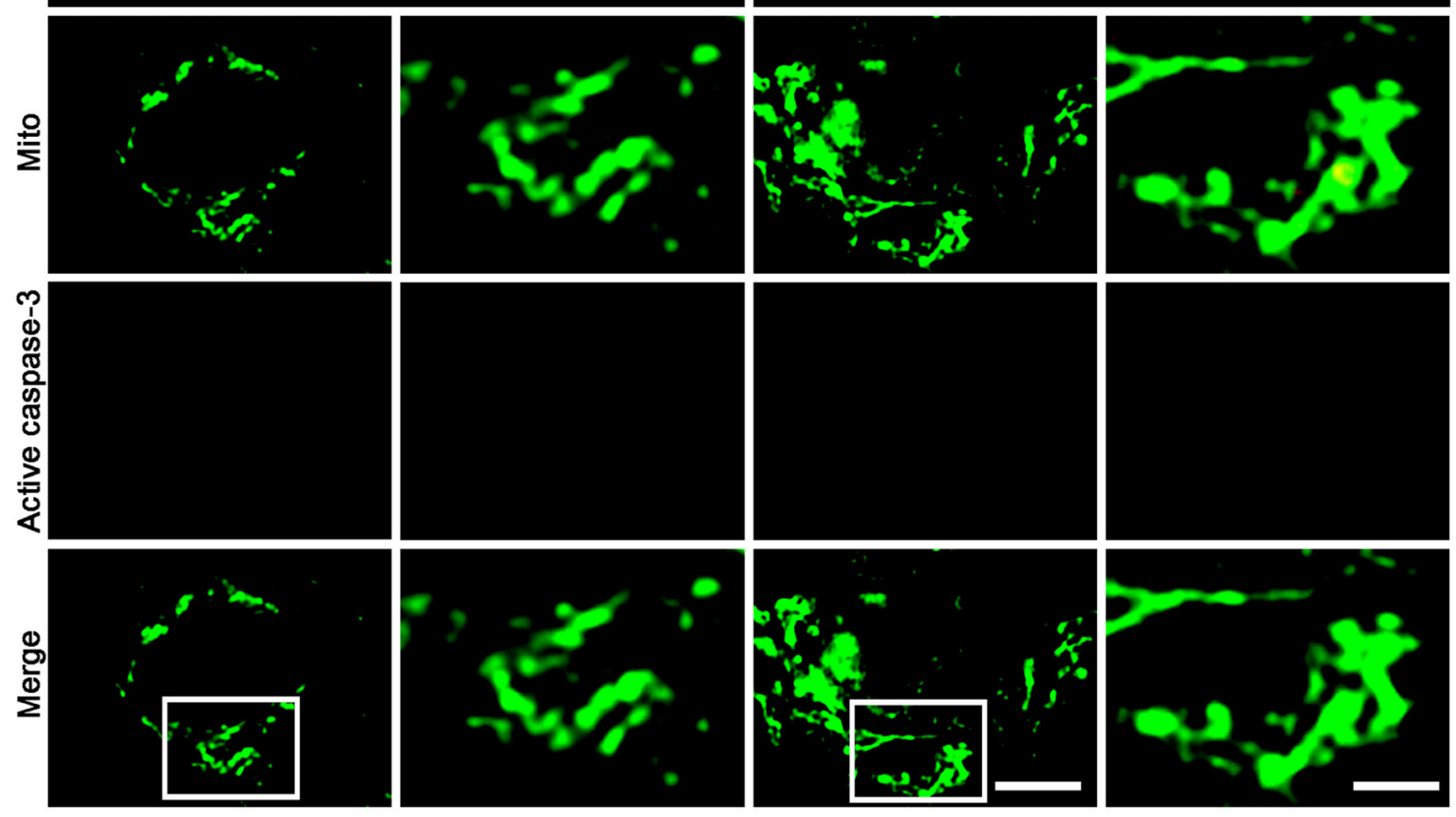

B
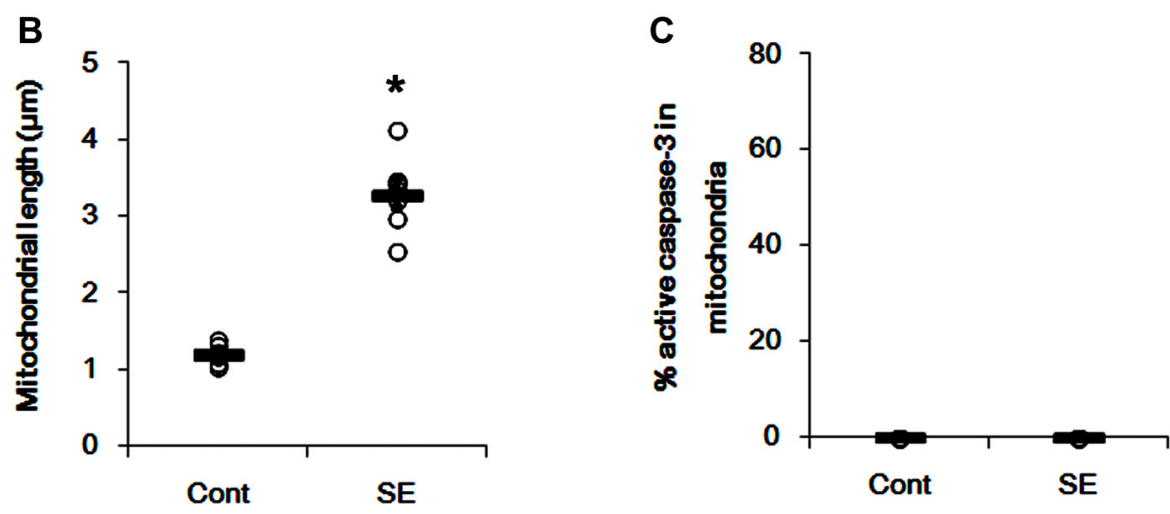

FIGURE 2 | Aberrant mitochondrial fusion in CA1 neurons 3 days after SE. SE results in mitochondrial elongation and sphere formation in CA1 neurons of vehicle-treated animals. Active caspase-3 is not transported into mitochondria. (A) Representative photos of mitochondria (Mito, green) and active caspase-3 (red) signals in CA1 neurons. Rectangles in merge panels (left) indicate the zoom areas for the high magnification photos (right). Bar = 5 (left panels) and 1.5 (right panels) $\mu \mathrm{m}$. (B,C) Quantification of the mitochondrial length (B) and the fraction of active caspase-3 positive mitochondria in total mitochondria (C) in CA1 neurons following SE. Open circles indicate each individual value. Horizontal bars indicate mean value. Error bars indicate SEM $\left({ }^{*} p<0.05\right.$ vs. control animals (Cont); $n=7$, respectively).

In control animals, WY14643 led mitochondrial fragmentation in CA1 neurons. It also induced mitochondrial active caspase- 3 imports in $\sim 19 \%$ of total mitochondria $(p<0.05$ vs. vehicle; Figures 6A-C). However, WY14643 did not induce CA1 neuronal death under physiological condition (Figures 6D,E). Following SE, WY14643 attenuated mitochondrial elongation in CA1 neurons $(p<0.05$ vs. vehicle; Figures 6A-C) and SE-induced CA1 neuronal death ( $p<0.05$ vs. vehicle; Figures 6D,E), although $\sim 18 \%$ of total mitochondria showed active caspase- 3 signals (Figures 6A-C). Taken together, these findings indicate that mitochondrial fission may play an important role in active caspase- 3 induction and its mitochondrial imports in PV cells and CA1 neurons under normal conditions, although it did not lead to cell death. Furthermore, it is likely that active caspase-3 transports into mitochondrial may be relevant to PV cell loss, but not CA1 neuronal death following SE.

\section{Mitochondrial HMGB1 Import in CA1 Neurons, Not PV Cell, Induced by SE}

In various cells, nucleocytopalsmic HMGB1 release is observed during necrosis (Scaffidi et al., 2002; Faraco et al., 2007; 

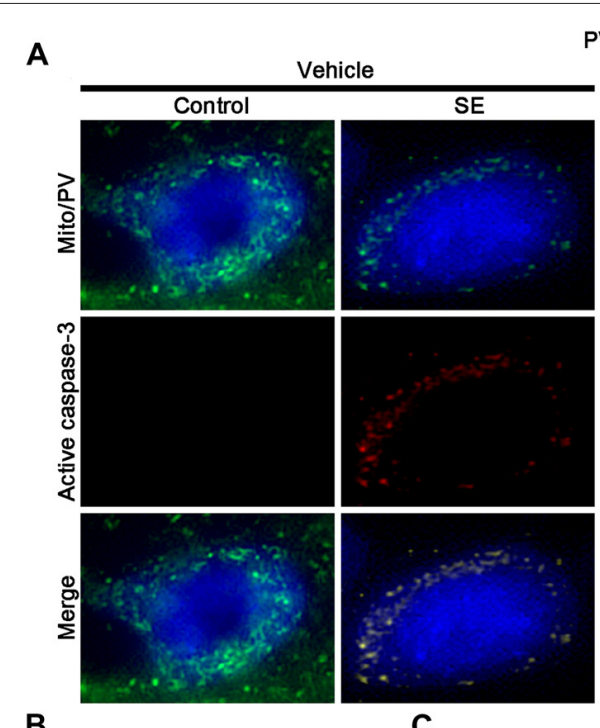

PV
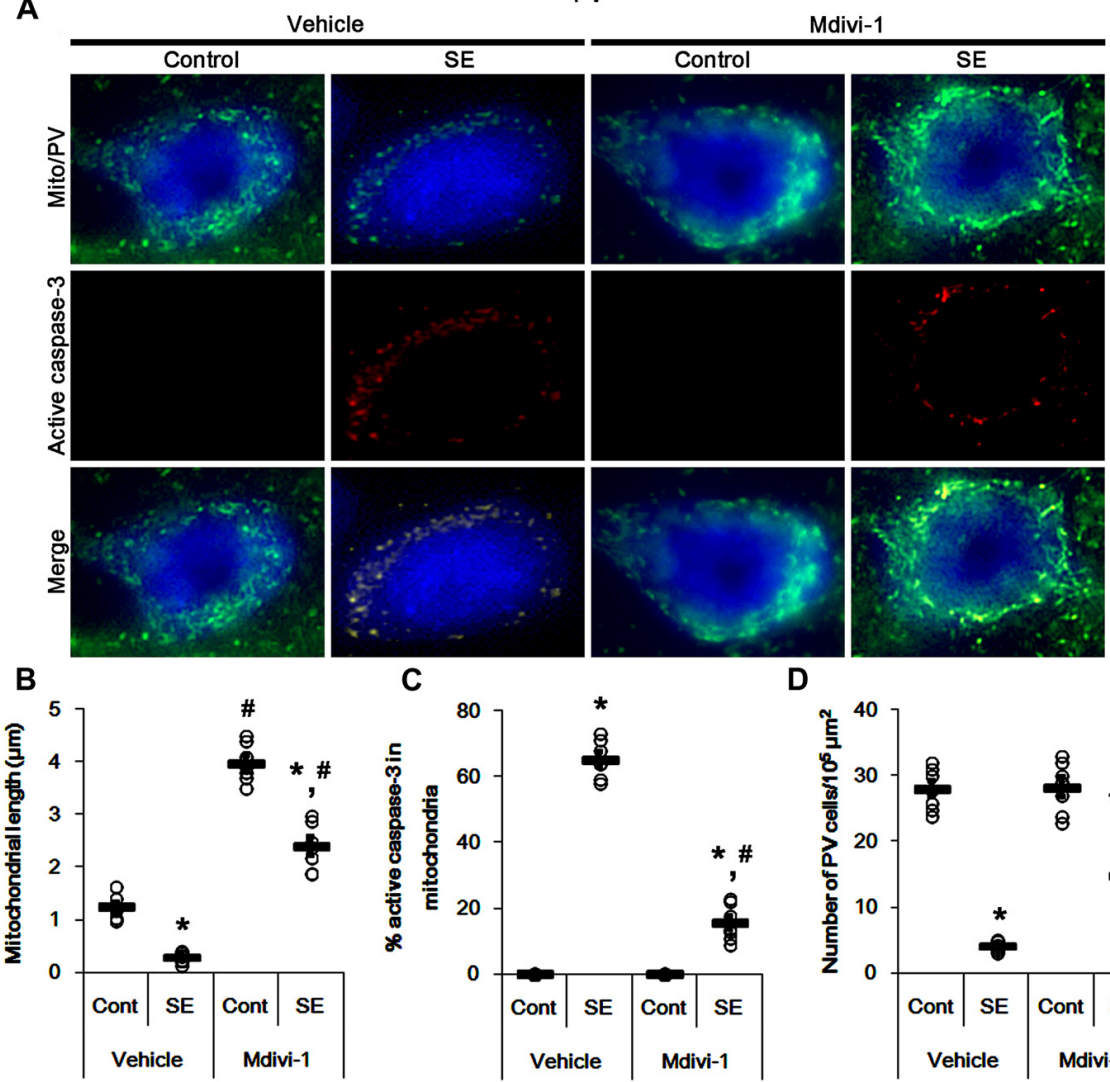

C
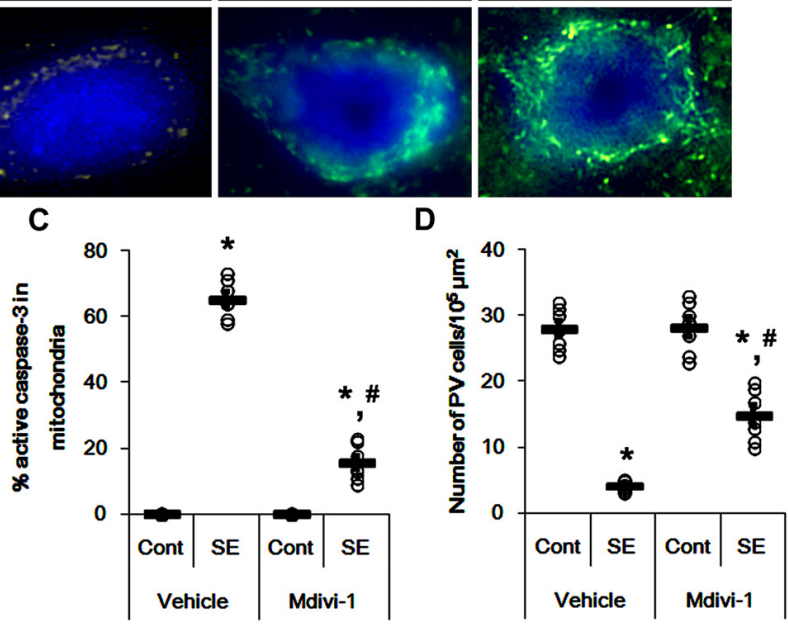

E

Vehicle

Mdivi-1
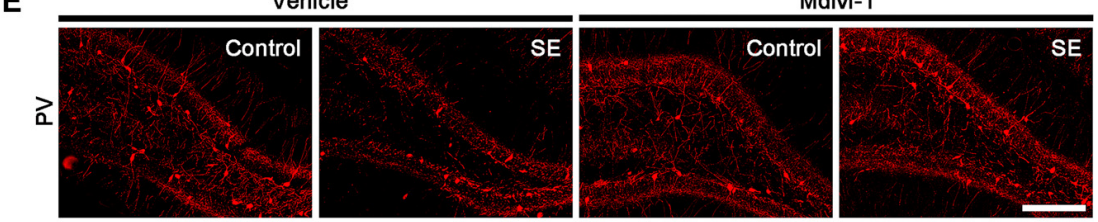

FIGURE 3 | Effects of Mdivi-1 on mitochondrial dynamics, active caspase-3 translocation and PV cell loss following SE. Mdivi-1 attenuates mitochondrial fragmentation, active caspase-3 transport into mitochondria and PV cell loss induced by SE. (A) Representative photos of mitochondria (Mito, green) and active caspase-3 (red) in PV (blue) cells. Bar $=5 \mu \mathrm{m}$. (B,C) Quantification of the mitochondrial length (B) and the fraction of active caspase-3 positive mitochondria in total mitochondria (C) in PV cells following SE. Open circles indicate each individual value. Horizontal bars indicate mean value. Error bars indicate SEM ( ${ }^{*}$, $p<0.05$ vs. control- (Cont) and vehicle-treated animals, respectively; $n=7$, respectively). (D) Quantification of the number of PV cells $12 \mathrm{~h}$ after SE. Open circles indicate each individual value. Horizontal bars indicate mean value. Error bars indicate SEM ${ }^{*},{ }^{\#} p<0.05$ vs. control- (Cont) and vehicle-treated animals; $n=7$, respectively).

(E) Representative photos of PV cells $12 \mathrm{~h}$ after SE. Bar $=100 \mu \mathrm{m}$.

Kim et al., 2014). Furthermore, translocation of HMGB1 to mitochondria may facilitate and deteriorate necrotic CA1 neuronal death (Hyun et al., 2016). Thus, we explored whether mitochondrial HMGB1 transports also influence SE-induced PV cell loss. In control animals, HMGB1 expression was restricted to the nuclei in PV cells. Six hours after SE, nuclear HMGB1 intensity was reduced in PV cells $(p<0.05$ vs. control; Figures 7A,B). However, few fragmented mitochondria contained HMGB1 signals in PV cells (Figures 7A,C). In CA1 neurons, SE induced HMGB1 signal in elongated mitochondria with reduction in nuclear HMGB1 level ( $p<0.05$ vs. control; Figures 8A-C).
These findings suggest that mitochondrial translocations of HMGB1 may be relevant to SE-induced necrotic CA1 neuronal death.

\section{SE-Induced Mitochondrial Elongation Accelerates Mitochondrial HMGB1 Translocation}

The remaining issue is whether mitochondrial dynamics also affect HMGB1 translocation to mitochondria in PV cells and CA1 neurons following SE. In the present study, Mdivi-1 and WY14643 did not lead to nuclear HMGB1 release in PV 
A

PV
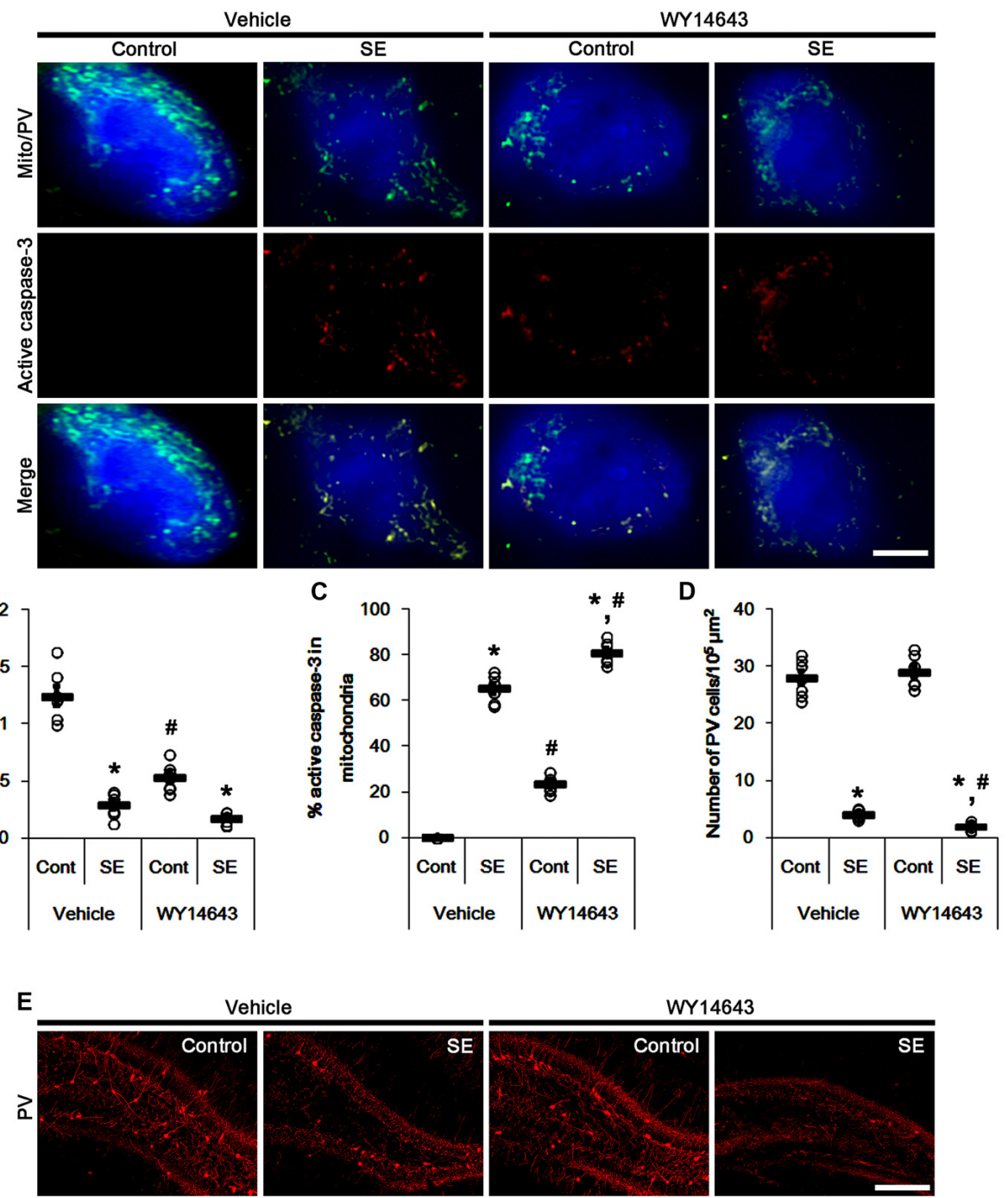

FIGURE 4 | Effects of WY14643 on mitochondrial dynamics, active caspase-3 translocation and PV cell loss following SE. WY14643 deteriorates mitochondrial fragmentation, mitochondrial active caspase-3 transport and PV cell loss induced by SE. (A) Representative photos of mitochondria (green) and active caspase-3 (red) in PV (blue) cells. Bar $=5 \mu \mathrm{m}$. (B,C) Quantification of the mitochondrial length (B) and the fraction of active caspase-3 positive mitochondria in total mitochondria (C) in PV cells following SE. Open circles indicate each individual value. Horizontal bars indicate mean value. Error bars indicate SEM (*, $p<<0.05$ vs. control-(Cont) and vehicle-treated animals, respectively; $n=7$, respectively). (D) Quantification of the number of PV cells $12 \mathrm{~h}$ after SE. Open circles indicate each individual value. Horizontal bars indicate mean value. Error bars indicate SEM $\left({ }^{*},{ }^{\#} p<0.05\right.$ vs. control-(Cont) and vehicle-treated animals, respectively; $n=7$, respectively). (E) Representative photos of PV cells $12 \mathrm{~h}$ after SE. Bar $=100 \mu \mathrm{m}$.

cells under physiological condition (Figures 9, 10). Mdivi-1 attenuated SE-induced nucleocytoplasmic HMGB1 translocation in PV cells ( $p<0.05$ vs. vehicle; Figures 9A,B). Mdivi-1 did not result in mitochondrial HMGB1 imports in PV cells of post-SE animals (Figures 9A,C). Although WY14643 could not abrogate nuclear HMGB1 release in PV cells (Figures 10A,B), it did not induce mitochondrial HMGB1 imports in PV cells of post-SE animals (Figures 10A,C).

Under normal conditions, both Mdivi-1 and WY14643 did not affect nuclear HMGB1 localization in CA1 neurons (Figures 11, 12). In post-SE animals, Mdivi-1 did not prevent nuclear HMGB1 export in CA1 neurons (Figures 11A,B). Furthermore, it enhanced SE-induced mitochondrial HMGB1 transport in CA1 neurons ( $p<0.05$ vs. vehicle; Figures 11A,C). However, WY14643 effectively attenuated nuclear HMGB1 release and its mitochondrial translocation ( $p<0.05$ vs. vehicle; Figures 12A-C). These findings indicate that mitochondrial dynamics may not participate in nuclear HMGB1 export and its mitochondrial translocation in both PV cells and CA1 neurons under normal conditions, but aberrant mitochondrial elongation may facilitate mitochondrial HMGB1 transport in CA1 neurons following SE. 


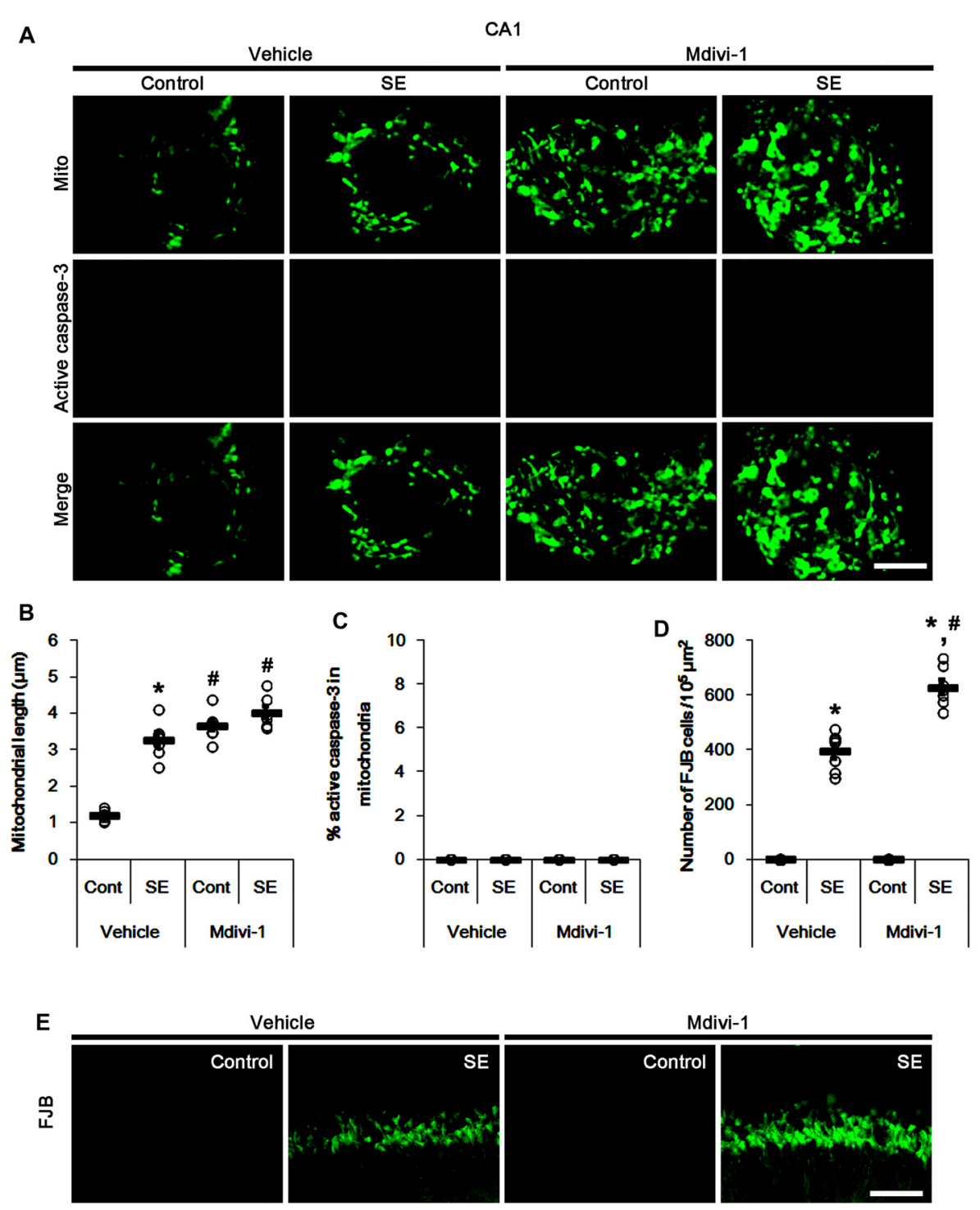

FIGURE 5 | Effects of Mdivi-1 on mitochondrial dynamics, active caspase-3 translocation and CA1 neuronal death following SE. Mdivi-1 aggravates mitochondrial elongation and CA1 neuronal damage without altered mitochondrial active caspase-3 transport 3 days after SE. (A) Representative photos of mitochondria (Mito, green) and active caspase-3 (red) in CA1 neurons. Bar $=5 \mu \mathrm{m}$. (B,C) Quantification of the mitochondrial length (B) and the fraction of active caspase-3 positive mitochondria in total mitochondria (C) in CA1 neurons following SE. Open circles indicate each individual value. Horizontal bars indicate mean value. Error bars indicate SEM (* ${ }^{\#} p<0.05$ vs. control-(Cont) and vehicle-treated animals, respectively; $n=7$, respectively). (D) Quantification of the number of fluoro-jade B (FJB) positive CA1 neurons following SE. Open circles indicate each individual value. Horizontal bars indicate mean value. Error bars indicate SEM $\left({ }^{*},{ }^{*} p<0.05\right.$ vs. control(Cont) and vehicle-treated animals, respectively; $n=7$, respectively). (E) Representative photos of FJB positive CA1 neurons 3 days after SE. Bar $=100 \mu m$.

\section{DISCUSSION}

Recently, we have reported that excessive mitochondrial fissions lead to apoptosis of PV cells in dentate gyrus following SE (Kim and Kang, 2017), while aberrant mitochondrial elongations evokes programed necrosis of CA1 neurons and dentate granule cells (Kim et al., 2014; Hyun et al., 2016; Ko and Kang, 2017). Consistent with these previous studies, the present data show that SE resulted in degenerations of PV cell and CA1 neurons accompanied by abnormal mitochondrial fission and fusion, respectively. Since mitochondrial dynamics are one of the important adaptive responses to the stressful stimuli (Chen and Chan, 2009; Rintoul and Reynolds, 2010), these findings indicate that the distinct impairment of mitochondrial dynamics may cause the different SE-induced cell death pattern between PV cells and CA1 neurons. However, the present study demonstrates that under physiological condition the inductions of mitochondrial fission or fusion by WY14643 and Mdivi-1 did not evoke neuronal death. Thus, it is likely that other factors may be involved in abnormal mitochondrial dynamics-mediated neuronal death under pathophysiological conditions. 
A

CA1

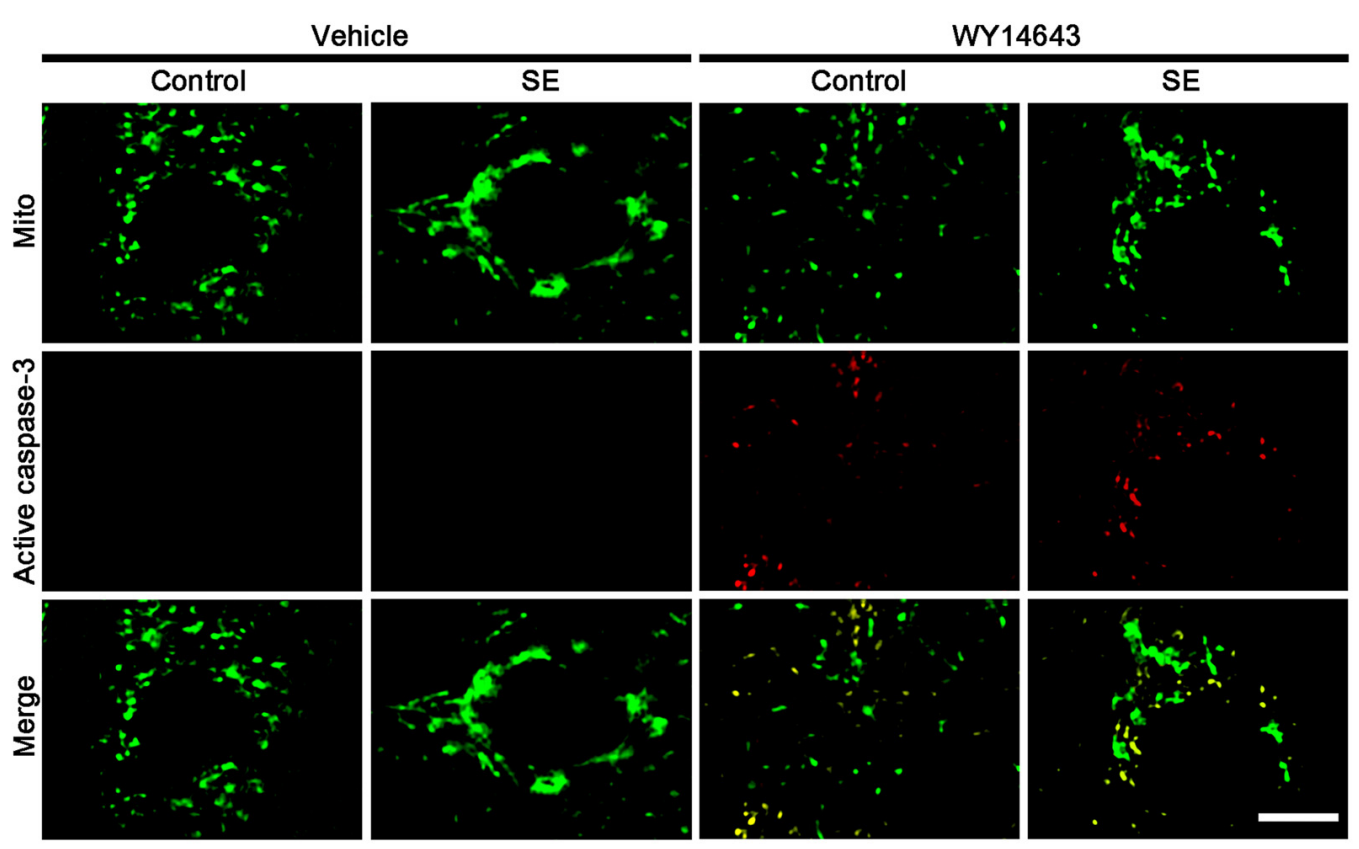

B

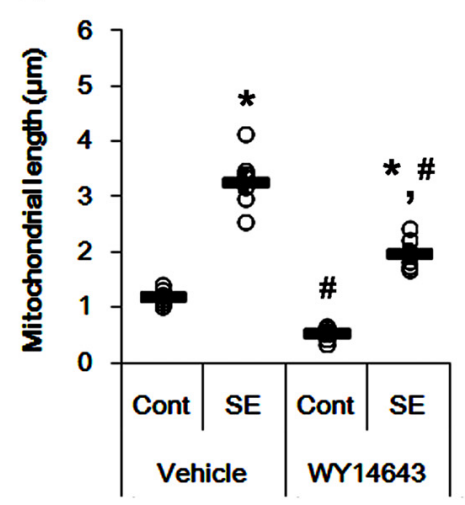

C

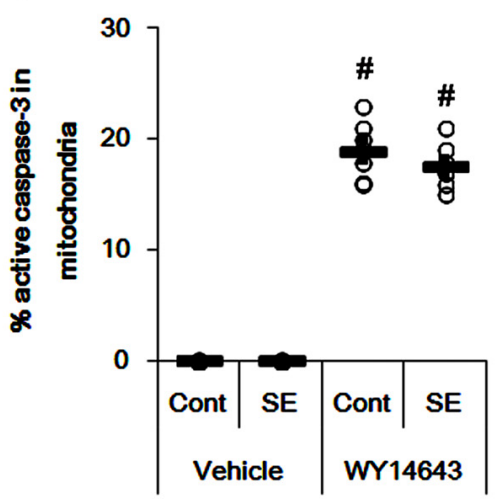

D

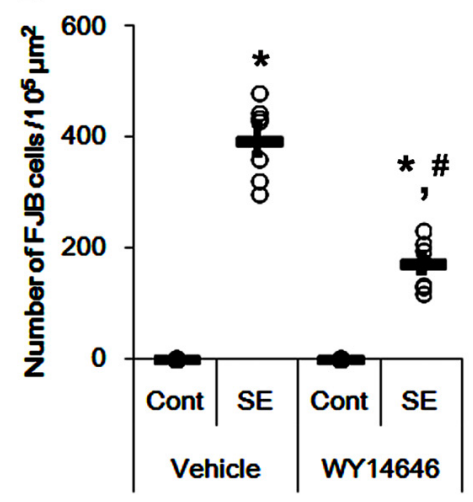

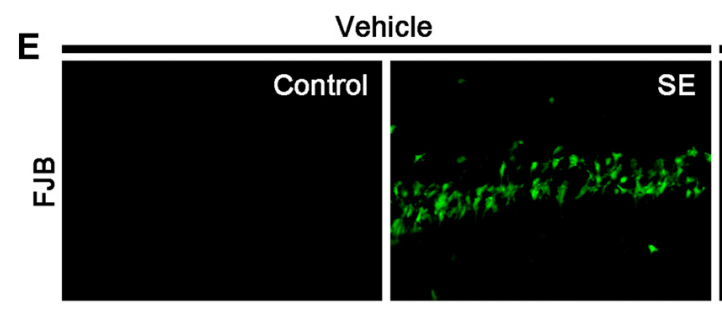

WY14643

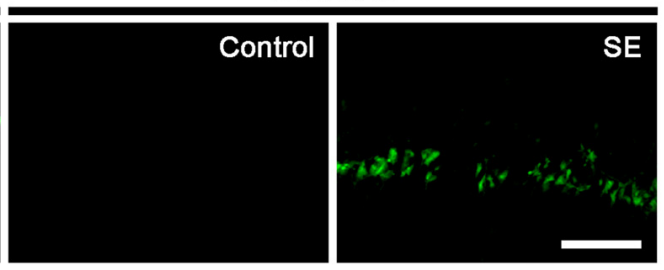

FIGURE 6 | Effects of WY14643 on mitochondrial dynamics, active caspase-3 translocation and CA1 neuronal death following SE. WY14643 induces mitochondrial fission and mitochondrial translocation of active caspase-3 in control animals. WY14643 ameliorates mitochondrial elongation and CA1 neuronal damage without altering active caspase-3 transport 3 days after SE. (A) Representative photos of mitochondria (Mito, green) and active caspase-3 (red) in CA1 neurons. Bar $=5 \mu \mathrm{m}$. $(\mathbf{B}, \mathbf{C})$ Quantification of the mitochondrial length (B) and the fraction of active caspase-3 positive mitochondria in total mitochondria (C) in CA1 neurons following SE. Open circles indicate each individual value. Horizontal bars indicate mean value. Error bars indicate SEM $\left({ }^{*}, \# p<0.05\right.$ vs. control- (Cont) and vehicle-treated animals, respectively; $n=7$, respectively). (D) Quantification of the number of FJB positive CA1 neurons following SE. Open circles indicate each individual value. Horizontal bars indicate mean value. Error bars indicate SEM ( ${ }^{*},{ }^{\circ} p<0.05$ vs. control- (Cont) and vehicle-treated animals, respectively; $n=7$, respectively). (E) Representative photos of FJB positive CA1 neurons 3 days after SE. Bar $=100 \mu \mathrm{m}$.

PV cell loss is one of the most acute and dramatic events induced by SE (Soukupová et al., 2014; Kim and
Kang, 2017). PV is one of the calcium-binding proteins, which is responsible for the fast-spiking capability of the 


\section{A}

PV
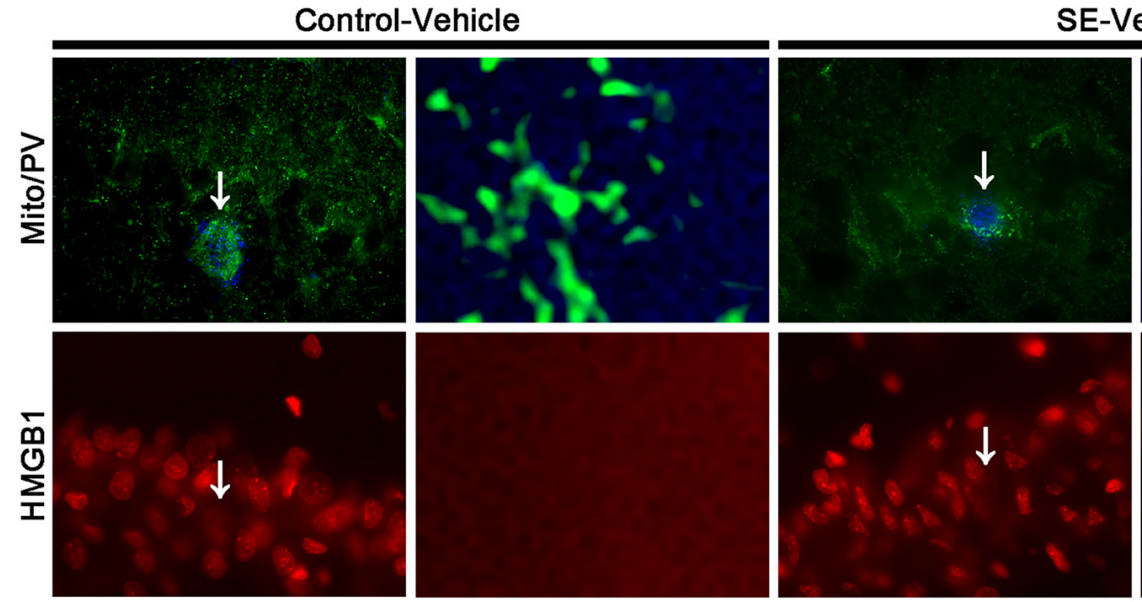

SE-Vehicle
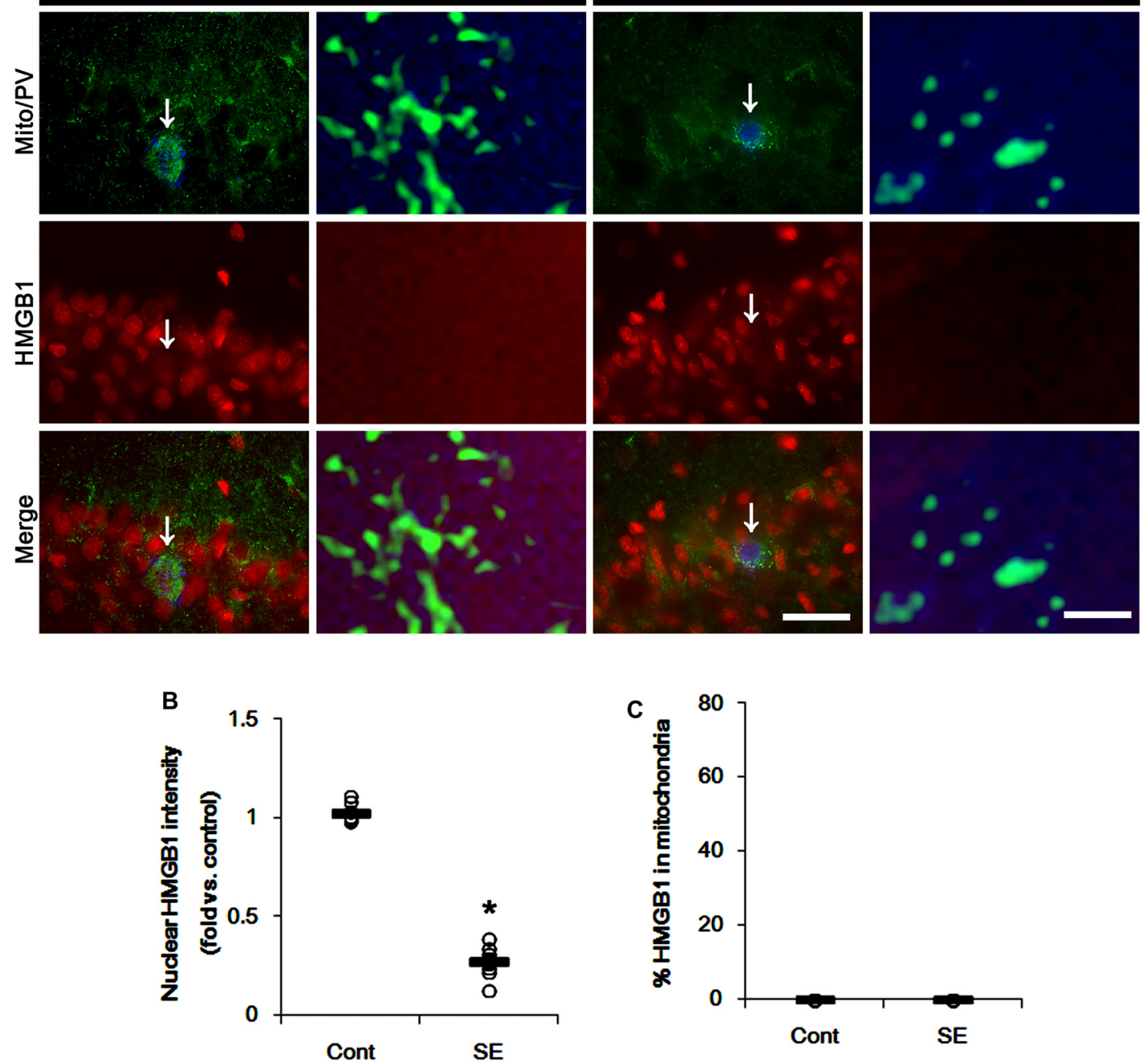

FIGURE 7 | Mitochondrial fission and nuclear High mobility group box 1 (HMGB1) release in PV cells $6 \mathrm{~h}$ after SE. SE rapidly induces mitochondrial fragmentation and nuclear HMGB1 release in PV cells of vehicle-treated animals. However, HMGB1 is not imported into mitochondria. (A) Representative photos of mitochondria (Mito, green) and HMGB1 (red) signals in PV (blue) cells. Arrows in left panels indicate the zoom areas for the high magnification of right panels. Bar = 25 (left panels) and 1.5 (right panels) $\mu \mathrm{m}$. (B,C) Quantification of the nuclear HMGB1 intensity (B) and the fraction of HMGB1 positive mitochondria in total mitochondria (C) in PV cells following SE. Open circles indicate each individual value. Horizontal bars indicate mean value. Error bars indicate SEM $\left({ }^{*} p<0.05\right.$ vs. control animals (Cont); $n=7$, respectively).

GABAergic neurons, which participate in a rapid adaptation in response to repetitive stimuli. Thus, PV cell loss leads to uncontrolled discharges and further epileptogenic processes (Sloviter, 1991; Sloviter et al., 2003; Cammarota et al., 2013; Elgueta et al., 2015). SE-induced PV cell death is caspase-3 dependent apoptosis concomitant with mitochondrial fragmentation (Kang et al., 2006; Kim and Kang, 2017). Excessive mitochondrial fission by enhancing dynamin-related proteins 1 (DRP1)-serine 616 phosphorylation impairs mitochondrial function and increases susceptibility to apoptotic stimuli (Campello and Scorrano, 2010; Kim and Kang, 2017). This is because the released cytochrome $c$ activates caspase-3 during mitochondrial fission (Frank et al., 2001; Breckenridge et al., 2003; Lee et al., 2004; Germain et al., 2005; Barsoum et al., 2006). Interestingly, the present study reveals the SE evoked massive active caspase- 3 translocation into mitochondria of PV cells, accompanied by the reduced mitochondrial length. Furthermore, WY14643 enhanced mitochondrial active caspase-3 transport and deteriorated PV cell degeneration induced by SE, which were mitigated by Mdivi-1. Since active caspase- 3 translocates into the mitochondria and disintegrates mitochondrial functions by degradation of mitochondrial 


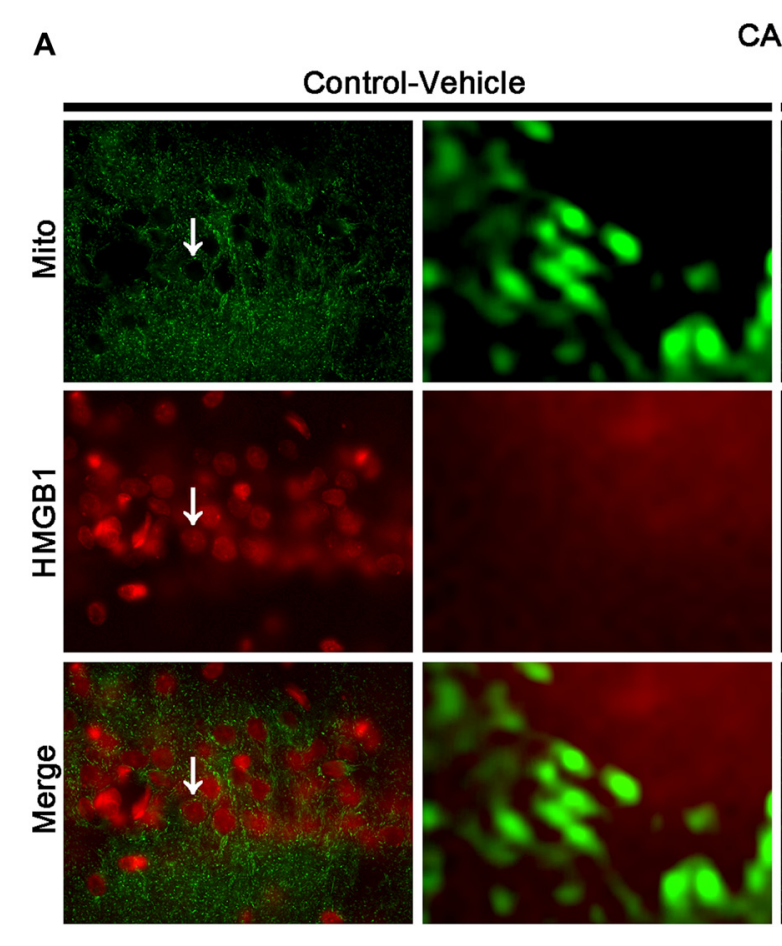

CA1

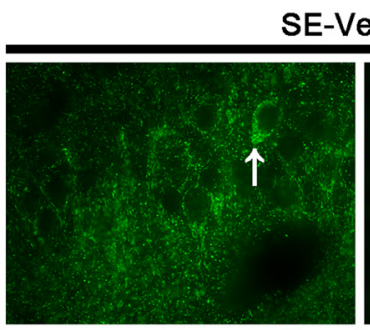

SE-Vehicle
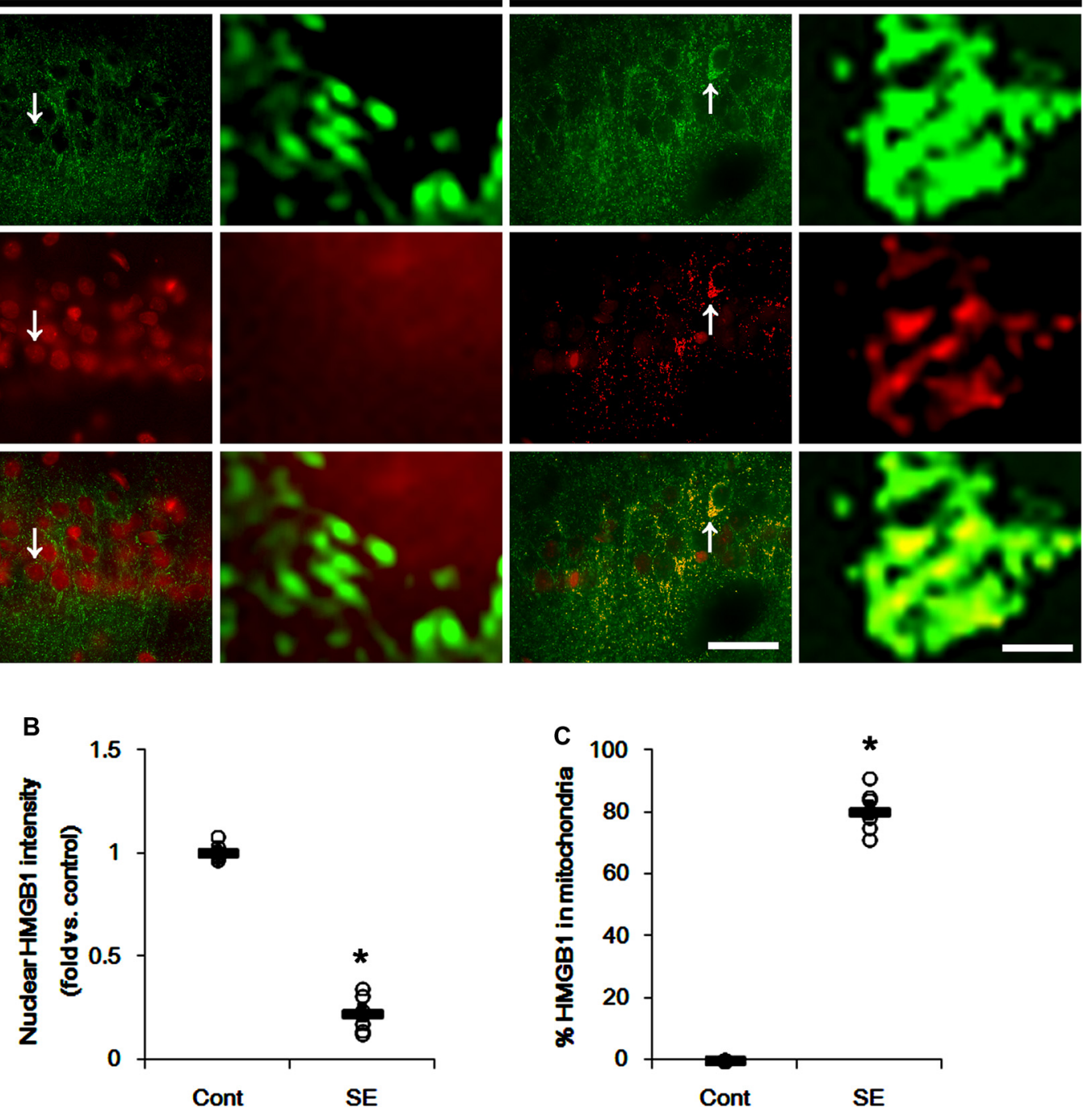

FIGURE 8 | Mitochondrial fusion and HMGB1 transport to mitochondria in CA1 neurons 3 days after SE. SE results in mitochondrial elongation and HMGB1 translocation from nuclei to mitochondria in CA1 neurons of vehicle-treated animals. (A) Representative photos of mitochondria (Mito, green) and HMGB1 (red) signals in CA1 neurons. Arrows in left panels indicate the zoom areas for the high magnification of right panels. Bar $=25$ (left panels) and 1.5 (right panels) $\mu \mathrm{m}$. (B,C) Quantification of the nuclear HMGB1 intensity (B) and the fraction of HMGB1 positive mitochondria in total mitochondria (B) in CA1 neurons following SE. Open circles indicate each individual value. Horizontal bars indicate mean value. Error bars indicate SEM $\left({ }^{*} p<0.05\right.$ vs. control animals (Cont); $n=7$, respectively).

constituents, especially in the late stage during apoptosis (Chandra and Tang, 2003), our findings suggest that preferential translocation of active caspase- 3 into mitochondria may facilitate SE-induced apoptosis in PV cells, accompanied by excessive mitochondrial fission.

Unlike PV cells, SE induces abnormal mitochondrial elongation in CA1 neurons, in turn provokes programed necrosis independent of caspase-3 activity (Kim et al., 2014; Hyun et al., 2016). Impaired mitochondrial fission (aberrant mitochondrial elongation) exerts improper segregations of mitochondria and impaired mitochondrial transports, which result in neuronal death by reducing bioenergetics and respiratory function in peripheral sites of neurons (Parone et al., 2008; DuBoff et al., 2012; Kageyama et al., 2012; Kim et al., 2014). In the present study, SE led to aberrant mitochondrial elongation in CA1 neurons. Furthermore, Mdivi-1 increased the HMGB1 transport into mitochondria in CA1 neurons, and aggravated SE-induced CA1 cell loss. Together with data concerning mitochondrial active caspase-3 translocation in PV cells, our findings suggest that active caspase-3 and HMGB1 imports into mitochondria may be one of the regulatory factors in abnormal mitochondrial machinery-mediated neuronal death following SE. 
A

PV
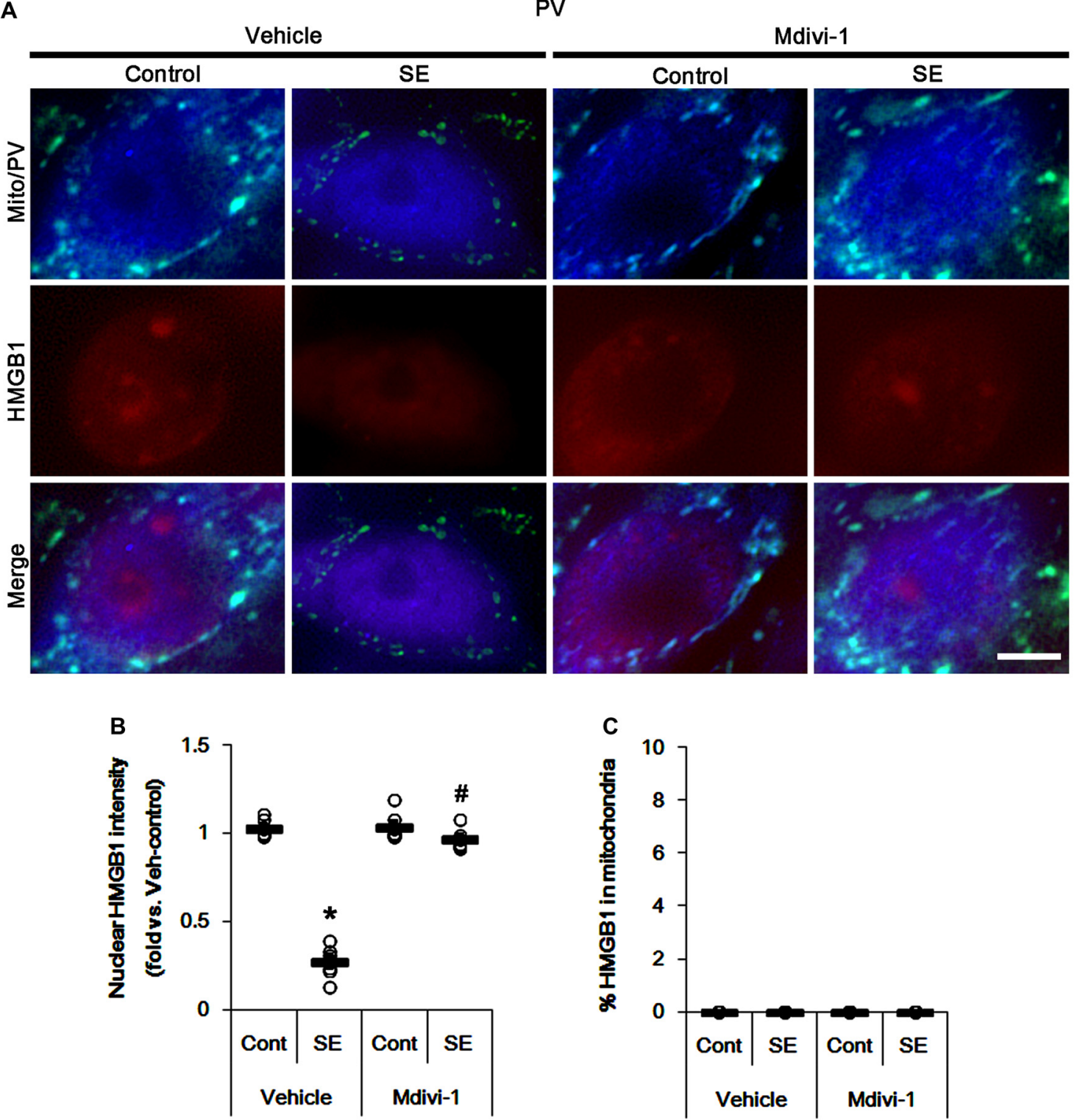

FIGURE 9 | Effects of Mdivi-1 on mitochondrial dynamics and HMGB1 translocation in PV cells $6 \mathrm{~h}$ after SE. Mdivi-1 attenuates mitochondrial fragmentation and nuclear HMGB1 release $6 \mathrm{~h}$ after SE. Mdivi-1 does not induce HMGB1 translocation to mitochondria. (A) Representative photos of mitochondria (Mito, green) and HMGB1 (red) in PV (blue) cells. Bar = $5 \mu \mathrm{m}$. (B,C) Quantification of the nuclear HMGB1 intensity (B) and the fraction of HMGB1 positive mitochondria in total mitochondria (C) in PV cells $6 \mathrm{~h}$ after SE. Open circles indicate each individual value. Horizontal bars indicate mean value. Error bars indicate SEM $\left(^{*}, \# p<0.05\right.$ vs. control- (Cont) and vehicle-treated animals, respectively; $n=7$, respectively).

Although nuclear HMGB1 release is an indicative of necrosis in various cells (Scaffidi et al., 2002; Faraco et al., 2007; Qiu et al., 2008), HMGB1 translocates into mitochondria and regulate their functions and reorganizations (Stumbo et al., 2008; Ito et al., 2014). Furthermore, translocation of HMGB1 into elongated mitochondria facilitates SE-induced CA1 neuronal death, while nuclear HMGB1 export could not affect mitochondrial dynamics (Hyun et al., 2016). In the present study, Mdivi-1 enhanced SE-induced mitochondrial HMGB1 transport in CA1 neurons, while was attenuated by WY14643. However, mitochondrial HMGB1 import was not observed in PV cell following SE, although nuclear HMGB1 release was detected. These findings indicate that the mitochondrial elongation may increase HMGB1 permeability into mitochondria. In addition, neither Mdivi-1 nor WY14643 resulted in mitochondrial HMGB1 transports in PV cells of control and post-SE animals. Therefore, our findings indicate that translocation of HMGB1 into mitochondria may be one of specific phenomena undergoing CA1 neuronal necrosis. 


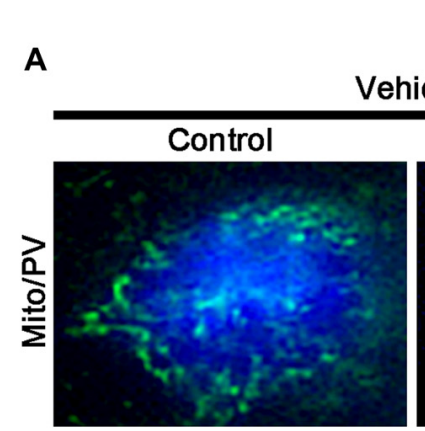

PV

A Vehicle WY14643
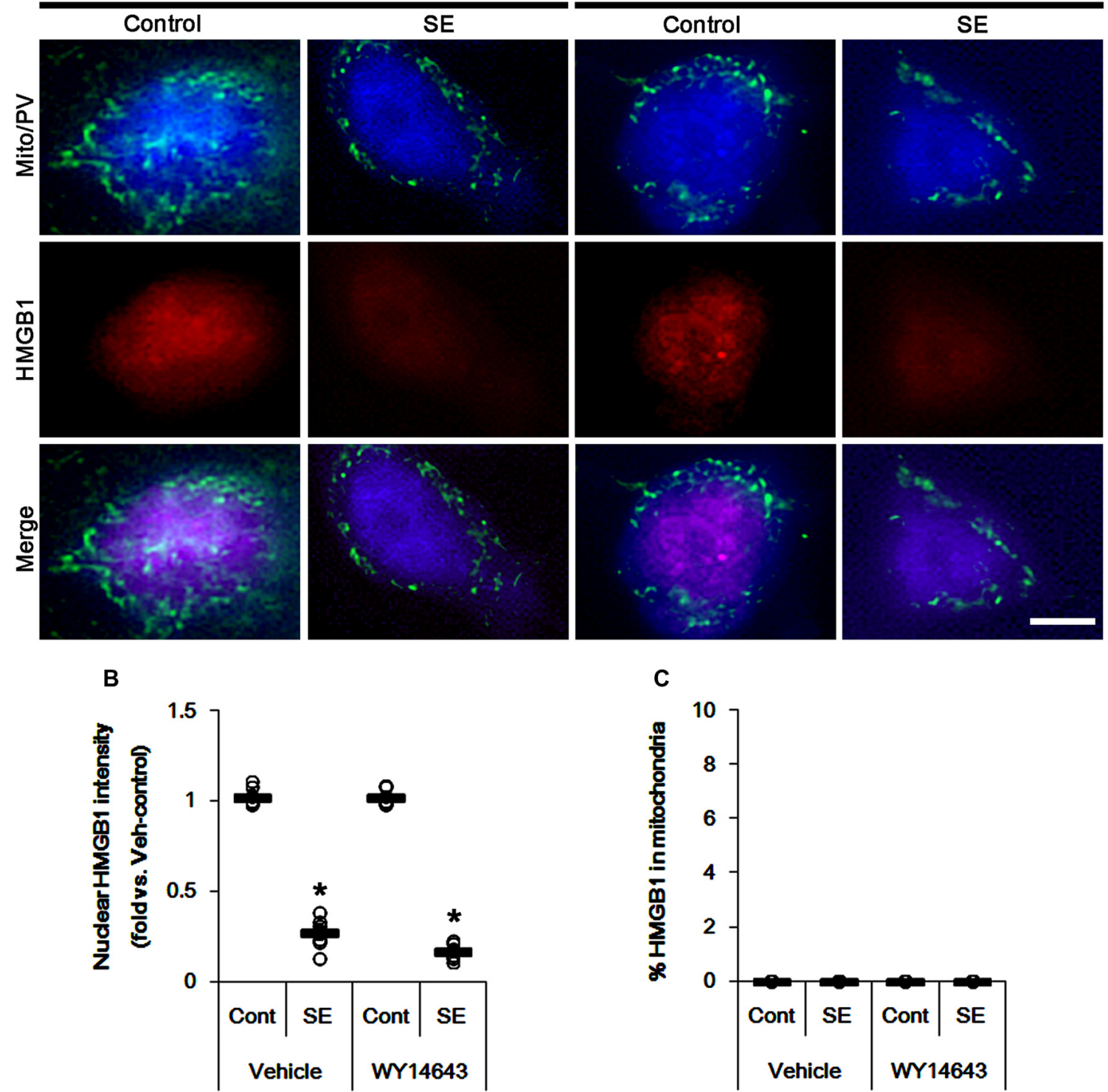

FIGURE 10 | Effects of WY14643 on mitochondrial dynamics and HMGB1 translocation in PV cells 6 h after SE. WY14643 does not affect mitochondrial fragmentation and nuclear HMGB1 release following SE. In addition, WY14643 does not induce mitochondrial HMGB1 translocation. (A) Representative photos of mitochondria (Mito, green) and HMGB1 (red) in PV (blue) cells. Bar = $5 \mu \mathrm{m}$. (B,C) Quantification of the nuclear HMGB1 intensity (B) and the fraction of HMGB1 positive mitochondria in total mitochondria (C) in PV cells $6 \mathrm{~h}$ after SE. Open circles indicate each individual value. Horizontal bars indicate mean value. Error bars indicate SEM ( ${ }^{*} p<0.05$ vs. control animals (Cont); $n=7$, respectively).

In the present study, under physiological condition WY14643 resulted in mitochondrial fissions and active caspase-3 translocations in $\sim 24 \%$ and $\sim 19 \%$ of total mitochondria of $\mathrm{PV}$ cells and CA1 neurons, respectively, although it did not induce the degenerations of these neurons. Since mitochondrial fission regulates a normal rate of cytochrome $c$ release (Ishihara et al., 2009), these findings indicate that the rate of active caspase- 3 into total mitochondria induced by WY14643 may be insufficient to evoke
PV-and CA1 neuronal death under physiological condition. Indeed, WY14643 attenuated SE-induced CA1 neuronal death, in spite of active caspase- 3 imports in $\sim 18 \%$ of total mitochondria. Following SE, furthermore, degenerating PV cell showed active caspase- 3 signals in $\sim 65 \%$ of total mitochondria, which were increased to $\sim 80 \%$ by WY14643. Taken together, our findings indicate that mitochondrial fission may be required for caspase- 3 activation under normal and pathophysiological conditions, and suggest that SE-induced 
Vehicle

A
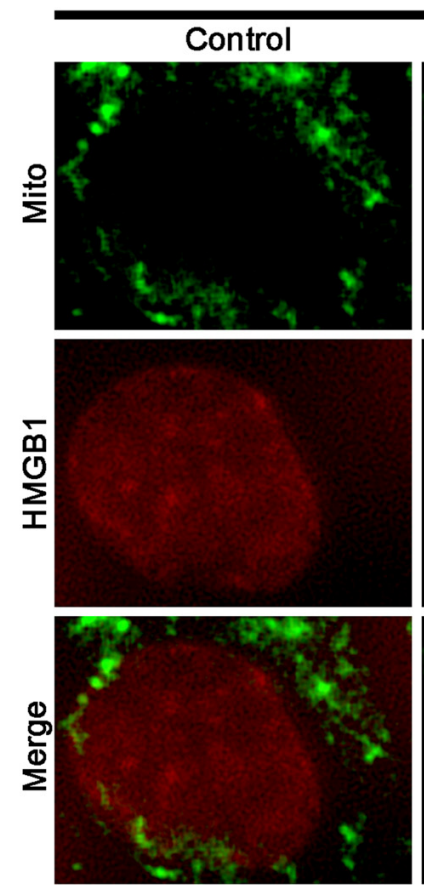

B

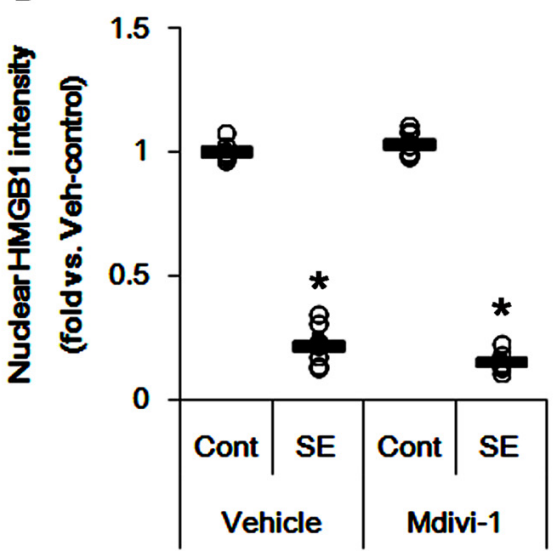

CA1

Mdivi-1
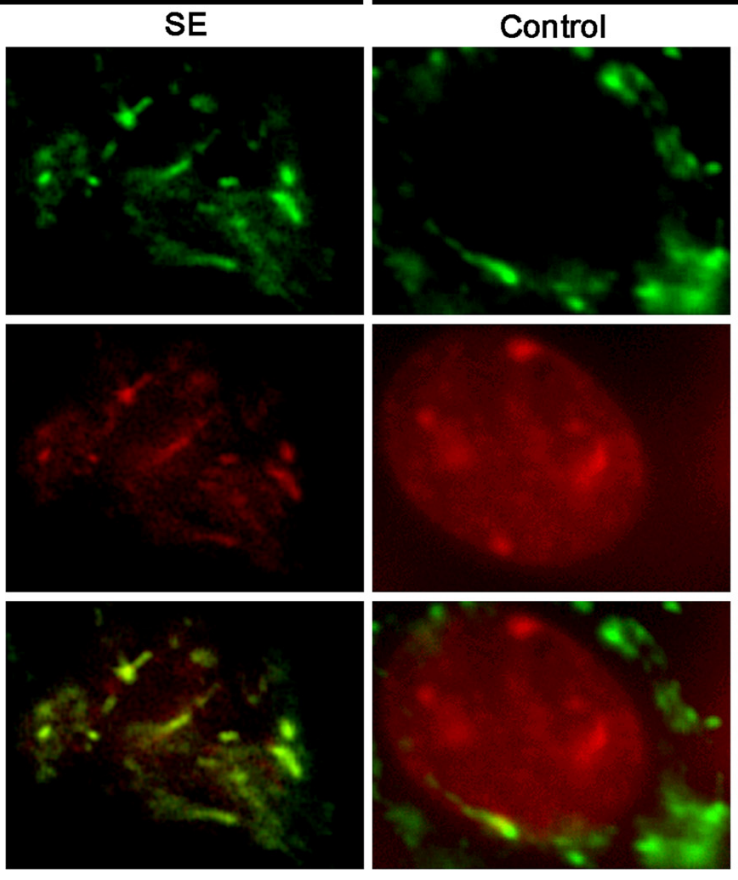

C

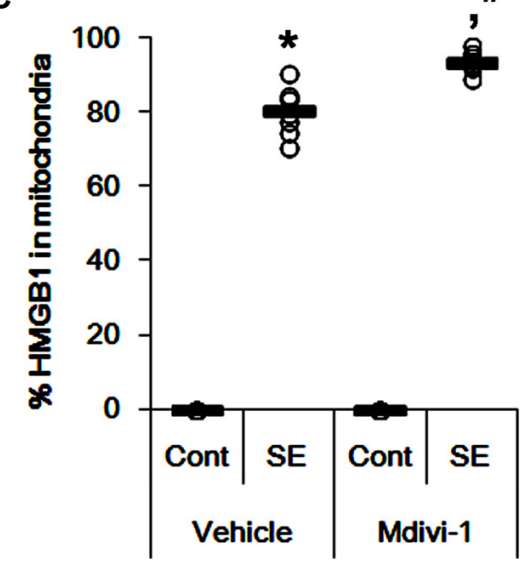

FIGURE 11 | Effects of Mdivi-1 on mitochondrial dynamics and HMGB1 translocation in CA1 neurons 3 days after SE. Mdivi-1 aggravates mitochondrial elongation and mitochondrial HMGB1 translocation in CA1 neurons following SE. (A) Representative photos of mitochondria (Mito, green) and HMGB1 (red) in CA1 neurons following SE. Bar $=5 \mu \mathrm{m}$. (B,C) Quantification of the nuclear HMGB1 intensity (B) and the fraction of HMGB1 positive mitochondria in total mitochondria (C) in CA1 neurons 3 days after SE. Open circles indicate each individual value. Horizontal bars indicate mean value. Error bars indicate SEM ${ }^{*},{ }^{\#} p<0.05$ vs. control(Cont) and vehicle-treated animals, respectively; $n=7$, respectively).

aberrant mitochondrial fusion in CA1 neurons may evoke necrosis rather than apoptosis due to inability of caspase-3 activation.

Why do PV cell and CA1 neurons show the distinct patterns of mitochondrial dynamics in response to pilocarpine-induced SE? We could not directly address this issue. Interestingly, the controversial effects of Mdivi-1 on SE-induced neuronal death would be considerable. Some reports demonstrate that Mdivi-1 attenuates neuronal loss after SE (Qiu et al., 2013; Xie et al., 2013), similar to the case of PV cells in the present study. However, the present study reveals that Mdivi-1 deteriorated SE-induced CA1 neuronal death. These discrepancies are resulted from the distinct methodology inducing SE: 1 h-lasting SE (Qiu et al., 2013; Xie et al., $2013)$ vs. 2 h-lasting models. Indeed, the differences in seizure activity lead to the distinct consequences on SE-induced 


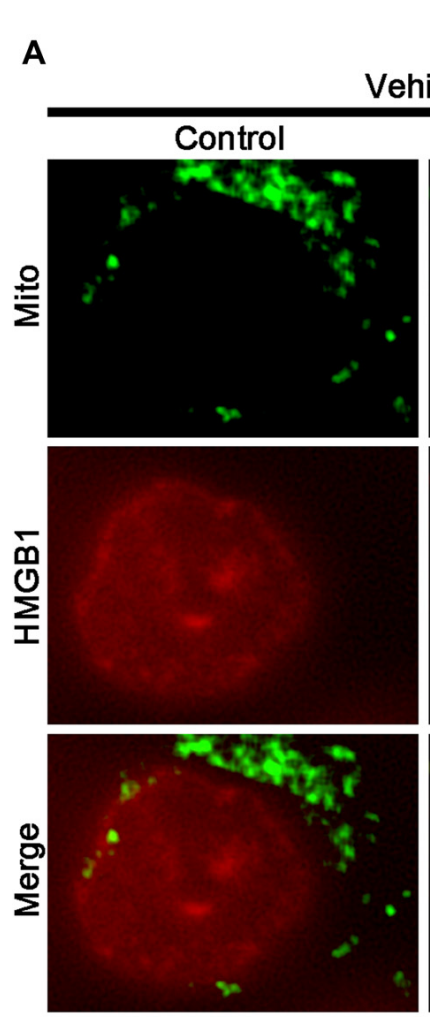

B

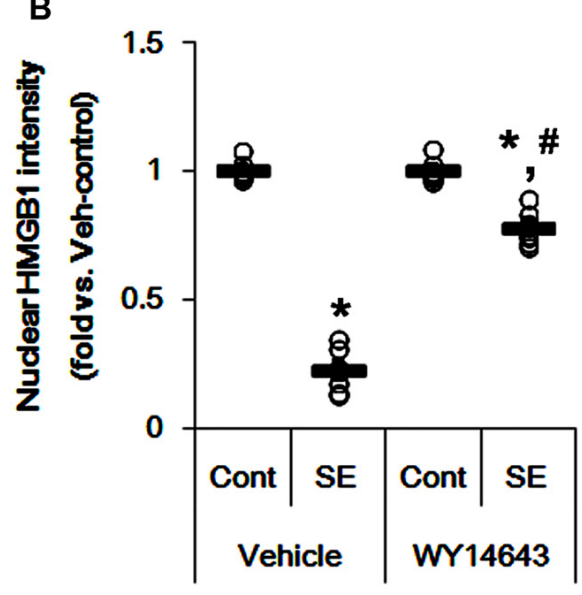

CA1

WY14643
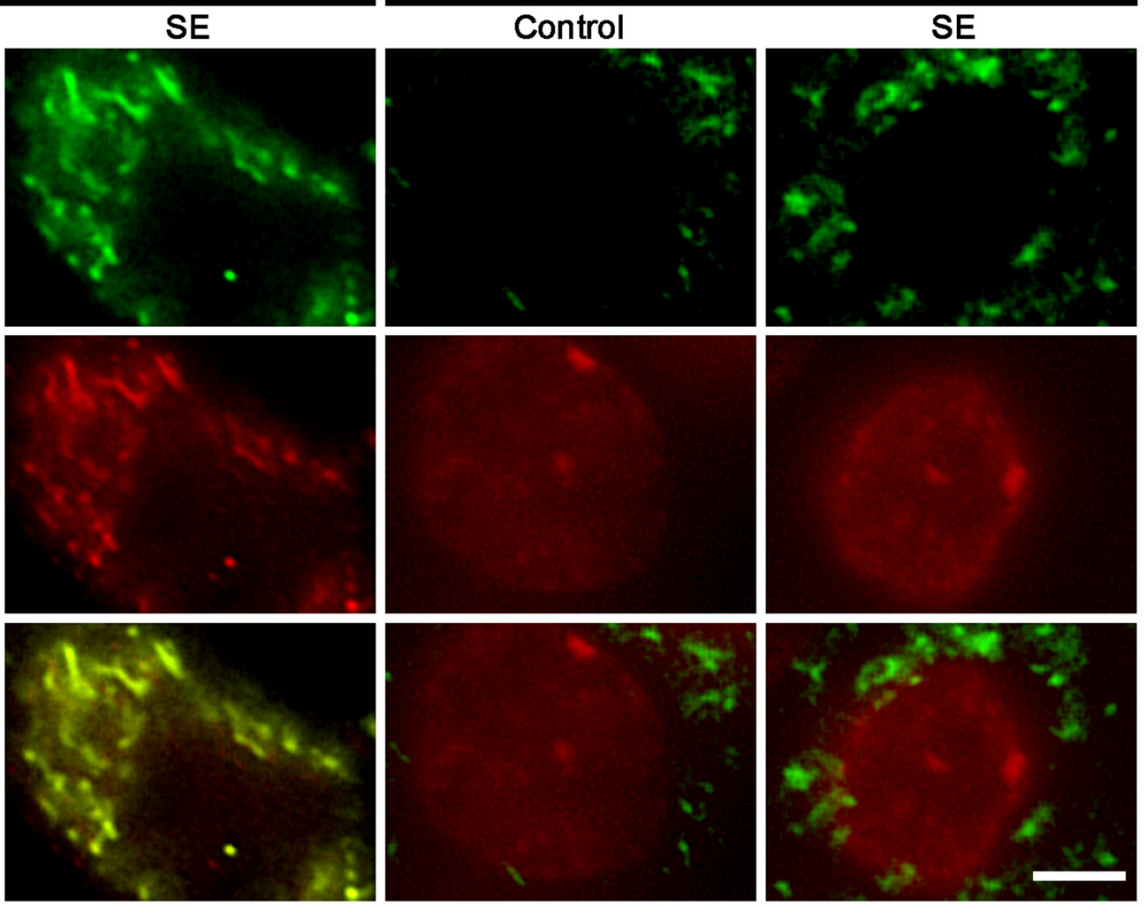

C

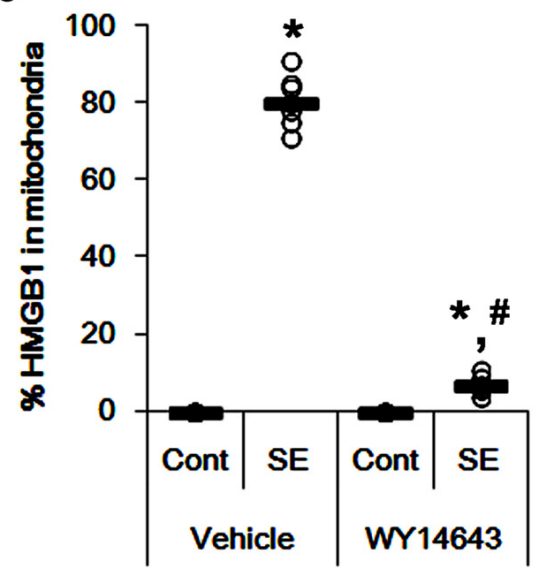

FIGURE 12 | Effects of WY14643 on mitochondrial dynamics and HMGB1 translocation in CA1 neurons 3 days after SE. WY14643 mitigates mitochondrial elongation and mitochondrial HMGB1 translocation in CA1 neurons following SE. (A) Representative photos of mitochondria (Mito, green) and HMGB1 (red) in CA1 neurons following SE. Bar $=5 \mu \mathrm{m}$. (B,C) Quantification of the nuclear HMGB1 intensity (B) and the fraction of HMGB1 positive mitochondria in total mitochondria (C) in CA1 neurons 3 days after SE. Open circles indicate each individual value. Horizontal bars indicate mean value. Error bars indicate SEM ( ${ }^{*}{ }^{\#} p<0.05$ vs. control- (Cont) and vehicle-treated animals, respectively; $n=7$, respectively).

neuronal death. Therefore, it is likely that the disparities in seizure susceptibility or firing rates during ictal stage may distinctly influence mitochondrial dynamics in different neuronal subpopulations. To validate this hypothesis, further studies are needed.

In conclusion, to the best of our knowledge, the present data provide the first evidence that SE-induced aberrant mitochondrial dynamics were involved in the mitochondrial translocations of active caspase- 3 and HMGB1 in PV cells and CA1 neurons, respectively. Furthermore, these phenomena were closely relevant to the differential cell death patterns of PV cell and CA1 neurons in response to SE. Therefore, the identification of mitochondrial permeable molecules and their preferential events will be interesting and considerable topics to understand the cell death mechanisms relevant to impaired mitochondrial dynamics. 


\section{AUTHOR CONTRIBUTIONS}

J-EK and T-CK designed the project. J-EK and T-CK performed the experiments described in the manuscript, analyzed the data and wrote the manuscript.

\section{REFERENCES}

Barsoum, M. J., Yuan, H., Gerencser, A. A., Liot, G., Kushnareva, Y., Graber, S., et al. (2006). Nitric oxide-induced mitochondrial fission is regulated by dynamin-related GTPases in neurons. EMBO J. 25, 3900-3911. doi: 10.1038/sj. emboj.7601253

Breckenridge, D. G., Stojanovic, M., Marcellus, R. C., and Shore, G. C. (2003). Caspase cleavage product of BAP31 induces mitochondrial fission through endoplasmic reticulum calcium signals, enhancing cytochrome $\mathrm{c}$ release to the cytosol. J. Cell Biol. 160, 1115-1127. doi: 10.1083/jcb.200212059

Cammarota, M., Losi, G., Chiavegato, A., Zonta, M., and Carmignoto, G. (2013). Fast spiking interneuron control of seizure propagation in a cortical slice model of focal epilepsy. J. Physiol. 591, 807-822. doi: 10.1113/jphysiol.2012.238154

Campello, S., and Scorrano, L. (2010). Mitochondrial shape changes: orchestrating cell pathophysiology. EMBO Rep. 11, 678-684. doi: 10.1038/embor. 2010.115

Chandra, D., and Tang, D. G. (2003). Mitochondrially localized active caspase-9 and caspase- 3 result mostly from translocation from the cytosol and partly from caspase-mediated activation in the organelle. J. Biol. Chem. 278, 17408-17420. doi: 10.1074/jbc.M300750200

Chen, H., and Chan, D. C. (2009). Mitochondrial dynamics-fusion, fission, movement, and mitophagy-in neurodegenerative diseases. Hum. Mol. Genet. 18, R169-R176. doi: 10.1093/hmg/ddp326

DuBoff, B., Götz, J., and Feany, M. B. (2012). Tau promotes neurodegeneration via DRP1 mislocalization in vivo. Neuron 75, 618-632. doi: 10.1016/j.neuron.2012. 06.026

Elgueta, C., Köhler, J., and Bartos, M. (2015). Persistent discharges in dentate gyrus perisoma-inhibiting interneurons require hyperpolarization-activated cyclic nucleotide-gated channel activation. J. Neurosci. 35, 4131-4139. doi: 10.1523/JNEUROSCI.3671-14.2015

Faraco, G., Fossati, S., Bianchi, M. E., Patrone, M., Pedrazzi, M., Sparatore, B., et al. (2007). High mobility group box 1 protein is released by neural cells upon different stresses and worsens ischemic neurodegeneration. J. Neurochem. 103, 590-603. doi: 10.1111/j.1471-4159.2007.04788.x

Frank, S., Gaume, B., Bergmann-Leitner, E. S., Leitner, W. W., Robert, E. G., Catez, F., et al. (2001). The role of dynamin-related protein 1, a mediator of mitochondrial fission, in apoptosis. Dev. Cell 1, 515-525. doi: 10.1016/s15345807(01)00055-7

Gdynia, G., Keith, M., Kopitz, J., Bergmann, M., Fassl, A., Weber, A. N., et al. (2010). Danger signaling protein HMGB1 induces a distinct form of cell death accompanied by formation of giant mitochondria. Cancer Res. 70, 8558-8568. doi: 10.1158/0008-5472.CAN-10-0204

Germain, M., Mathai, J. P., McBride, H. M., and Shore, G. C. (2005). Endoplasmic reticulum BIK initiates DRP1-regulated remodelling of mitochondrial cristae during apoptosis. EMBO J. 24, 1546-1556. doi: 10.1038/sj.emboj.7600592

Hyun, H. W., Ko, A. R., and Kang, T. C. (2016). Mitochondrial translocation of high mobility group box 1 facilitates LIM kinase 2-mediated programmed necrotic neuronal death. Front. Cell. Neurosci. 10:99. doi: 10.3389/fncel.2016. 00099

Ishihara, N., Nomura, M., Jofuku, A., Kato, H., Suzuki, S. O., Masuda, K., et al. (2009). Mitochondrial fission factor Drp1 is essential for embryonic development and synapse formation in mice. Nat. Cell Biol. 11, 958-966. doi: $10.1038 /$ ncb1907

Ito, H., Fujita, K., Tagawa, K., Chen, X., Homma, H., Sasabe, T., et al. (2014). HMGB1 facilitates repair of mitochondrial DNA damage and extends the lifespan of mutant ataxin-1 knock-in mice. EMBO Mol. Med. 7, 78-101. doi: 10.15252/emmm.201404392

Kageyama, Y., Zhang, Z., Roda, R., Fukaya, M., Wakabayashi, J., Wakabayashi, N., et al. (2012). Mitochondrial division ensures the survival of postmitotic neurons by suppressing oxidative damage. J. Cell Biol. 197, 535-551. doi: 10.1083/jcb. 201110034

\section{FUNDING}

This study was supported by a grant of National Research Foundation of Korea (NRF) grant (No. 2018R1A2A2A05018222).

Kang, T. C., Kim, D. S., Kwak, S. E., Kim, J. E., Won, M. H., Kim, D. W., et al. (2006). Epileptogenic roles of astroglial death and regeneration in the dentate gyrus of experimental temporal lobe epilepsy. Glia 54, 258-271. doi: $10.1002 /$ glia.20380

Kim, J. E., and Kang, T. C. (2017). p47Phox/CDK5/DRP1-mediated mitochondrial fission evokes pv cell degeneration in the rat dentate gyrus following status epilepticus. Front. Cell. Neurosci. 11:267. doi: 10.3389/fncel.2017.00267

Kim, J. E., Ryu, H. J., and Kang, T. C. (2011). P2X7 receptor activation ameliorates CA3 neuronal damage via a tumor necrosis factora-mediated pathway in the rat hippocampus following status epilepticus. J. Neuroinflammation 8:62. doi: $10.1186 / 1742-2094-8-62$

Kim, J. E., Ryu, H. J., Kim, M. J., and Kang, T. C. (2014). LIM kinase-2 induces programmed necrotic neuronal death via dysfunction of DRP1-mediated mitochondrial fission. Cell Death Differ. 21, 1036-1049. doi: 10.1038/cdd. 2014.17

Ko, A. R., Hyun, H. W., Min, S. J., and Kim, J. E. (2016). The differential DRP1 phosphorylation and mitochondrial dynamics in the regional specific astroglial death induced by status epilepticus. Front. Cell. Neurosci. 10:124. doi: $10.3389 /$ fncel.2016.00124

Ko, A. R., Hyun, H. W., Min, S. J., Kim, J. E., and Kang, T. C. (2015). Endothelin-1 induces LIMK2-mediated programmed necrotic neuronal death independent of NOS activity. Mol. Brain 8:58. doi: 10.1186/s13041-015-0149-3

Ko, A. R., and Kang, T. C. (2017). TRPC6-mediated ERK1/2 phosphorylation prevents dentate granule cell degeneration via inhibiting mitochondrial elongation. Neuropharmacology 121, 120-129. doi: 10.1016/j.neuropharm. 2017.05.004

Lee, Y. J., Jeong, S. Y., Karbowski, M., Smith, C. L., and Youle, R. J. (2004). Roles of the mammalian mitochondrial fission and fusion mediators Fis1, Drp1, and Opa1 in apoptosis. Mol. Biol. Cell 15, 5001-5011. doi: 10.1091/mbc.e04 -04-0294

Lundgren, B., Bergstrand, A., Karlsson, K., and DePierre, J. W. (1990). Effects of dietary treatment with clofibrate, nafenopin or WY-14.643 on mitochondria and DNA in mouse liver. J. Biol. Chem. 1035, 132-138. doi: 10.1016/03044165(90)90107-8

Mathern, G. W., Babb, T. L., Vickrey, B. G., Melendez, M., and Pretorius, J. K. (1995). The clinical-pathogenic mechanisms of hippocampal neuron loss and surgical outcomes in temporal lobe epilepsy. Brain 118, 105-118. doi: 10.1093/brain/118.1.105

Ordy, J. M., Wengenack, T. M., Bialobok, P., Coleman, P. D., Rodier, P., Baggs, R. B., et al. (1993). Selective vulnerability and early progression of hippocampal CA1 pyramidal cell degeneration and GFAP-positive astrocyte reactivity in the rat four-vessel occlusion model of transient global ischemia. Exp. Neurol. 119, 128-139. doi: 10.1006/exnr.1993.1014

Parone, P. A., Da Cruz, S., Tondera, D., Mattenberger, Y., James, D. I., Maechler, P., et al. (2008). Preventing mitochondrial fission impairs mitochondrial function and leads to loss of mitochondrial DNA. PLoS One 3:e3257. doi: 10.1371/journal.pone.0003257

Qiu, X., Cao, L., Yang, X., Zhao, X., Liu, X., Han, Y., et al. (2013). Role of mitochondrial fission in neuronal injury in pilocarpine-induced epileptic rats. Neuroscience 245, 157-165. doi: 10.1016/j.neuroscience.2013. 04.019

Qiu, J., Nishimura, M., Wang, Y., Sims, J. R., Qiu, S., Savitz, S. I., et al. (2008). Early release of HMGB-1 from neurons after the onset of brain ischemia. J. Cereb. Blood Flow Metab. 28, 927-938. doi: 10.1038/sj.jcbfm.9600582

Rintoul, G. L., and Reynolds, I. J. (2010). Mitochondrial trafficking and morphology in neuronal injury. Biochim. Biophys. Acta 1802, 143-150. doi: 10.1016/j.bbadis.2009.09.005

Ryu, H. J., Kim, J. E., Yeo, S. I., Kim, M. J., Jo, S. M., and Kang, T. C. (2011). ReLA/P65-serine 536 nuclear factor-kappa B phosphorylation is related to vulnerability to status epilepticus in the rat hippocampus. Neuroscience 187, 93-102. doi: 10.1016/j.neuroscience.2011.04.048 
Scaffidi, P., Misteli, T., and Bianchi, M. E. (2002). Release of chromatin protein HMGB1 by necrotic cells triggers inflammation. Nature 418, 191-195. doi: 10.1038/nature00858

Sloviter, R. S. (1991). Permanently altered hippocampal structure, excitability and inhibition after experimental status epilepticus in the rat: the "dormant basket cell" hypothesis and its possible relevance to temporal lobe epilepsy. Hippocampus 1, 41-66. doi: 10.1002/hipo.450010106

Sloviter, R. S., Zappone, C. A., Harvey, B. D., Bumanglag, A. V., Bender, R. A., and Frotscher, M. (2003). "Dormant basket cell" hypothesis revisited: relative vulnerabilities of dentate gyrus mossy cells and inhibitory interneurons after hippocampal status epilepticus in the rat. J. Comp. Neurol. 459, 44-76. doi: 10.1002/cne.10630

Soukupová, M., Binaschi, A., Falcicchia, C., Zucchini, S., Roncon, P., Palma, E., et al. (2014). Impairment of GABA release in the hippocampus at the time of the first spontaneous seizure in the pilocarpine model of temporal lobe epilepsy. Exp. Neurol. 257, 39-49. doi: 10.1016/j.expneurol.2014.04.014

Stumbo, A. C., Cortez, E., Rodrigues, C. A., Henriques, M. D., Porto, L. C., Barbosa, H. S., et al. (2008). Mitochondrial localization of nonhistone protein HMGB1 during human endothelial cell-Toxoplasma gondii infection. Cell Biol. Int. 32, 235-238. doi: 10.1016/j.cellbi.2007.08.031

Suen, D. F., Norris, K. L., and Youle, R. J. (2008). Mitochondrial dynamics and apoptosis. Genes Dev. 22, 1577-1590. doi: 10.1101/gad.1658508

Wittner, L., Maglóczky, Z., Borhegyi, Z., Halász, P., Tóth, S., Eross, L., et al. (2001). Preservation of perisomatic inhibitory input of granule cells in the epileptic human dentate gyrus. Neuroscience 108, 587-600. doi: 10.1016/s03064522(01)00446-8

Xie, N., Wang, C., Lian, Y., Zhang, H., Wu, C., and Zhang, Q. (2013). A selective inhibitor of Drp1, mdivi-1, protects against cell death of hippocampal neurons in pilocarpine-induced seizures in rats. Neurosci. Lett. 545, 64-68. doi: 10.1016/j.neulet.2013.04.026

Youle, R. J., and Karbowski, M. (2005). Mitochondrial fission in apoptosis. Nat. Rev. Mol. Cell Biol. 6, 657-663. doi: 10.1038/nrm1697

Zolezzi, J. M., Silva-Alvarez, C., Ordenes, D., Godoy, J. A., Carvajal, F. J., Santos, M. J., et al. (2013). Peroxisome proliferator-activated receptor (PPAR) $\gamma$ and $\operatorname{PPAR} \alpha$ agonists modulate mitochondrial fusion-fission dynamics: relevance to reactive oxygen species (ROS)-related neurodegenerative disorders. PLoS One 8:e64019. doi: 10.1371/journal.pone.0064019

Conflict of Interest Statement: The authors declare that the research was conducted in the absence of any commercial or financial relationships that could be construed as a potential conflict of interest.

Copyright (c) $2018 \mathrm{Kim}$ and Kang. This is an open-access article distributed under the terms of the Creative Commons Attribution License (CC BY). The use, distribution or reproduction in other forums is permitted, provided the original author(s) and the copyright owner(s) are credited and that the original publication in this journal is cited, in accordance with accepted academic practice. No use, distribution or reproduction is permitted which does not comply with these terms. 